Aus der Neurologischen Klinik und Poliklinik der Ludwig-Maximilians-Universität München Direktorin: Prof. Dr. med. Marianne Dieterich

\title{
Bildgebende Diagnostik der Epilepsien
}

\author{
Kumulative Habilitationsschrift \\ zur Erlangung der Venia Legendi im Fach \\ Experimentelle Neurologie
}

vorgelegt von

Dr. med. Christian Vollmar

2018 


\section{Inhaltsverzeichnis}

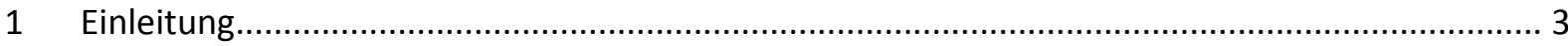

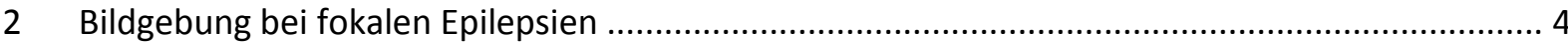

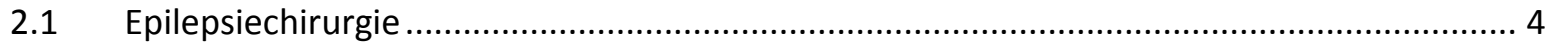

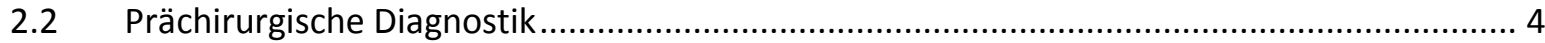

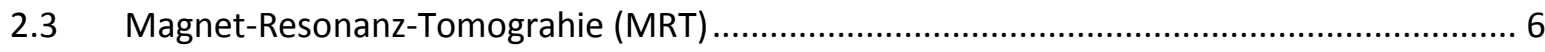

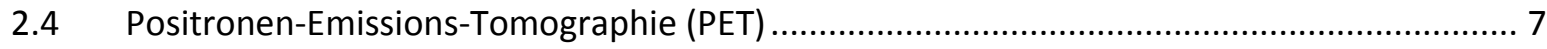

2.5 Single-Photon-Emissions-Computer-Tomographie (SPECT) .............................................. 7

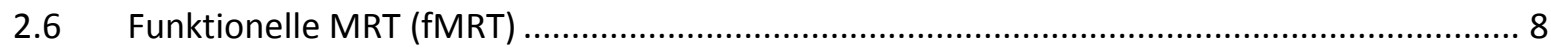

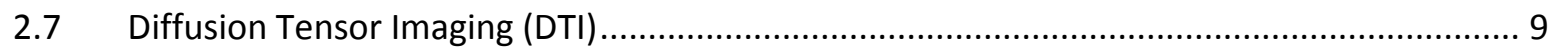

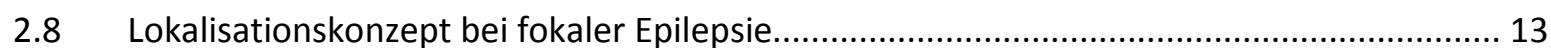

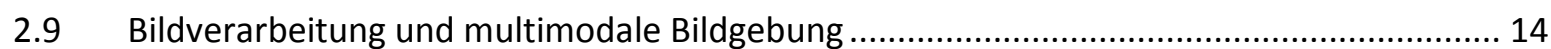

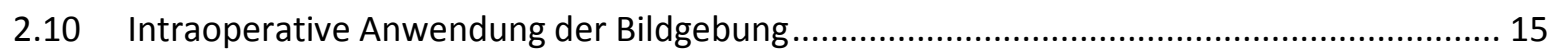

2.11 Ergebnisse nach epilepsiechirurgischen Eingriffen ............................................................. 17

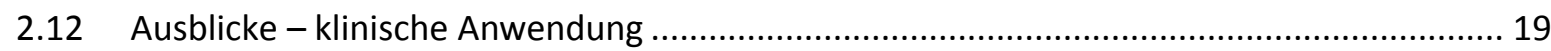

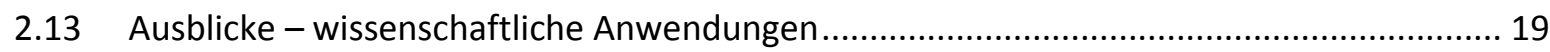

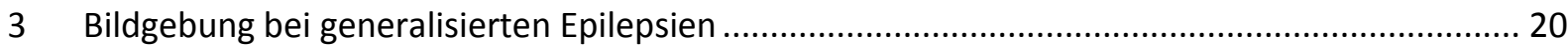

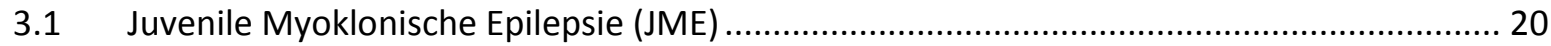

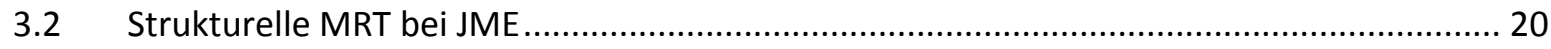

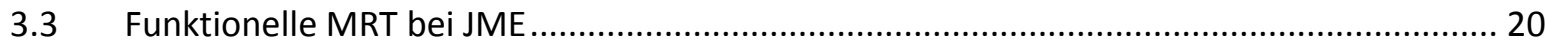

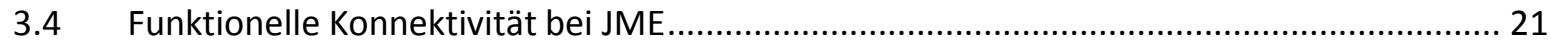

3.5 DTI und strukturelle Konnektivität bei JME ...................................................................... 22

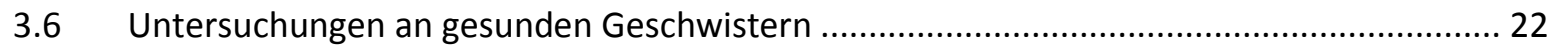

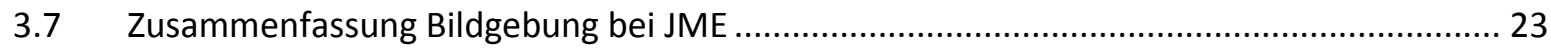

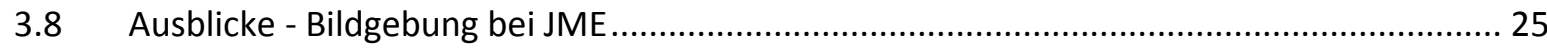

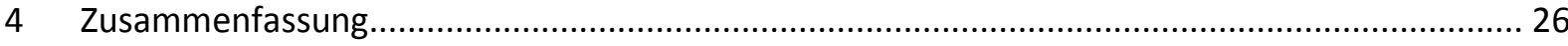

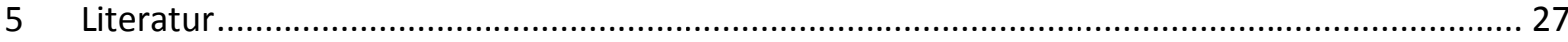

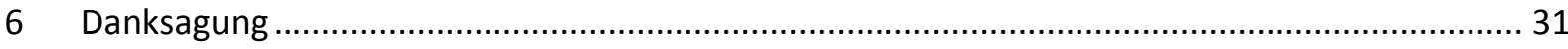

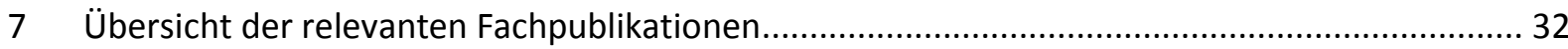




\section{Einleitung}

Epilepsie ist eine der häufigsten, schweren neurologischen Erkrankungen, von der knapp ein Prozent der Bevölkerung betroffen ist und die eine hohe sozioökonomische Belastung darstellt (de Boer et al., 2008). Neben den akuten Auswirkungen epileptischer Anfälle leiden Patienten häufig unter Komorbiditäten wie einer Depression, den Nebenwirkungen einer medikamentösen Behandlung und einer immer noch vorhandenen gesellschaftlichen Stigmatisierung. Langfristig entstehen im Verlauf der chronischen Erkrankung oft kognitive Defizite bis hin zur Berufsunfähigkeit und Frühberentung. Lebensqualität und die Lebenserwartung sind reduziert.

Die Klassifikation der Epilepsien unterscheidet im Wesentlichen zwischen generalisierten und fokalen Epilepsien. Bei generalisierten Epilepsien betrifft die zugrundeliegende Pathologie beide Hemisphären des Gehirns und auch zu Beginn eines Anfalles sind initial beide Hemisphären betroffen. Bei fokalen Epilepsien dagegen beginnt der epileptische Anfall in einer umschriebenen Region in nur einer Hemisphäre, kann sich jedoch im weiteren Verlauf ausbreiten, auch in die kontralaterale Hemisphäre. Diese Klassifikation ist klinisch wichtig und erlaubt eine bessere Prognoseabschätzung, eine Auswahl der möglichen Therapieoptionen und innerhalb des Spektrums der medikamentösen Behandlung die Auswahl des optimalen Mittels.

Die medikamentöse Behandlung der Epilepsien ist die wichtigste Therapieform, die auch bei etwa zwei Drittel der Patienten erfolgreich ist und zur dauerhaften Anfallsfreiheit führt. Insbesondere die generalisierten Epilepsien sprechen in der Regel gut auf eine medikamentöse Therapie an und viele Patienten sind unter einer gut verträglichen Medikation über viele Jahre oder Jahrzehnte anfallsfrei.

Bei dem verbleibenden Drittel der Patienten besteht jedoch Pharmakoresistenz, d.h. trotz teilweise hochdosierter Behandlung mit mehreren Antiepileptika treten weiterhin regelmäßig Anfälle auf. Patienten mit einer pharmakoresistenten fokalen Epilepsie sind potentiell Kandidaten für einen epilepsiechirurgischen Eingriff, bei dem das fokale Areal, von dem die Anfälle ihren Ursprung nehmen, neurochirurgisch entfernt wird, um die Anfälle zu stoppen. 


\section{Bildgebung bei fokalen Epilepsien}

Bei fokalen Epilepsien ist das Ziel der bildgebenden Diagnostik vorrangig die Identifikation der epileptogenen Läsion, die ursächlich für die Anfälle verantwortlich ist. Dies dient einer Klärung der Ätiologie der fokalen Epilepsie und erlaubt damit eine korrekte Syndromdefinition.

Eine besondere Bedeutung bekommt die Bildgebung, bei Patienten mit pharmakoresistenter fokaler Epilepsie, die sich mit der Frage nach einem möglichen epilepsiechirurgischen Eingriff vorstellen (Vollmar and Noachtar, 2004; Vollmar and la Fougère, 2012; Duncan et al., 2016).

\subsection{Epilepsiechirurgie}

Das Grundprinzip der Epilepsiechirurgie beruht darauf, den Teil des Gewebes, von dem die Anfälle ausgehen, durch einen neurochirurgischen Eingriff zu entfernen.

Gesundes Gehirngewebe generiert keine epileptischen Anfälle. Das heißt im Umkehrschluss, dass das Gewebe, das Anfälle generiert, pathologisch verändert ist - und daher in der Regel auch keine normalen Funktionen mehr ausführt. Deshalb ist dann auch eine Resektion dieses Areals ohne postoperatives Defizit möglich.

Epilepsiechirurgie ist eine hochwirksame Therapieoption für viele Patienten und ist die Therapie der Wahl für bestimmte Epilepsie-Syndrome. Bei einer mesialen Temporallappenepilepsie auf dem Boden einer Hippokampussklerose zum Beispiel beträgt die Chance, mit medikamentöser Behandlung dauerhaft anfallsfrei zu werden, nur etwa 8\%. Ein chirurgischer Eingriff mit Resektion des epileptogenen Areals führt dagegen in 58\% zu langfristiger Anfallsfreiheit (Wiebe et al., 2001). In den letzten Jahren berichten die meisten epilepsiechirurgischen Zentren von Anfallsfreiheits-Raten zwischen 30 und 70\% der operierten Patienten (Ryvlin et al., 2014; West et al., 2016). Die Erfolgsaussichten sind in der Regel für temporale Resektionen höher als für extratemporale Eingriffe.

Die individuelle Chance auf Anfallsfreiheit eines einzelnen Patienten hängt von multiplen Faktoren ab, die im Rahmen der prächirurgischen Evaluation untersucht werden.

\subsection{Prächirurgische Diagnostik}

Ziel der prächirurgischen Epilepsiediagnostik ist es, die Anfallsursprungszone zu lokalisieren, ihre Ausdehnung abzugrenzen und gleichzeitig zu prüfen, ob die Anfallsursprungszone ausreichend weit von eloquentem Kortex entfernt ist und damit eine Resektion gefahrlos möglich ist. Die drei wesentlichen Säulen der prächirurgischen Diagnostik sind:

1) Das Elektroencephalogramm (EEG).

Als ambulant durchgeführte Untersuchung kann das EEG zwischen den Anfällen interiktale epilepsietypische Potentiale aufzeichnen und lokalisieren und somit die Diagnose einer Epilepsie sichern und erste lokalisatorische Hinweise liefern. Im Rahmen einer Video-EEG-Monitoring Untersuchung werden Patienten über ein bis zwei Wochen rund um die Uhr mit Video und EEG überwacht. So kann auch das iktale EEG während des epileptischen Anfalles aufgezeichnet werden. Das EEG hat oft eine wichtige, lokalisatorische Bedeutung, allerdings sind die Befunde nicht immer konsistent, sondern können in manchen Fällen auch falsche Hinweise liefern. Insbesondere bei extratemporalen Epilepsien finden sich häufig EEG Befunde, die nicht die Region 
des Anfallsursprunges reflektieren (J Rémi et al., 2011) . In bestimmten anatomischen Konstellationen, kann es durch ungünstige Projektion elektrischer Felder zu einer falschen Lateralisierung von EEG Befunden kommen (Catarino et al., 2012).

2) Die klinisch-semiologische Information.

Anamnestische Angaben oder die klinische Beobachtung des Patienten während eines epileptischen Anfalles im Rahmen einer Video-EEG-Monitoring Untersuchung können ebenfalls wertvolle lokalisatorische Informationen liefern. Die Symptome, die im Verlauf eines epileptischen Anfalles auftreten, reflektieren die Funktion des zu diesem Zeitpunkt durch den Anfall aktivierten oder in seiner Funktion gestörten Hirnareals, der sogenannten symptomatogenen Zone. Eine epileptische Aktivierung des frontalen Augenfeldes führt beispielsweise zu einer Blick- und Kopfwendung zur kontralateralen Seite (Jan Rémi et al., 2011). Eine iktale oder postiktale Aphasie weist auf einen Anfallsursprung in der sprachdominanten Hemisphäre hin (Loesch et al., 2017). Bestehen bei einem Patienten verschiedene klinische Anfallsformen, kann dies auf unterschiedliche, evtl. bilaterale Anfallsursprünge hinweisen (Loesch et al., 2015). Da eine visuelle Beurteilung einiger klinischer Phänomene subjektiv und somit potentiell vom Befunder abhängig und nicht gut reproduzierbar ist, haben wir verschiedenen Methoden zur objektiven Quantifizierung von motorischen Phänomenen im 2D Videobild (Silva Cunha et al., 2013) oder aus einem 3D Video (Cunha et al., 2016) entwickelt, die benutzerunabhängige Kennzahlen zur Anfallssemiologie liefern können.

3) Die bildgebende Diagnostik, auf die im Folgenden noch genauer eingegangen wird.

Ein Grundprinzip der prächirurgischen Evaluation ist es, möglichst viele unabhängige diagnostische Methoden zu kombinieren. Weisen alle Methoden konsistent auf die gleiche Region hin, ist ein epilepsiechirurgischer Eingriff mit hoher Erfolgsaussicht möglich.

In einigen Fällen muss zunächst noch bestätigt werden, ob es sich bei den geschilderten Symptomen wirklich um epileptische Anfälle handelt (Vollmar and Noachtar, 2017), und nicht etwa um psychogene oder kardiale Ursachen (Vollmar et al., 2007).

Im Laufe der letzten Jahrzehnte hat sich in den meisten größeren Epilepsiezentren das Patientenspektrum verändert. Während früher meist relativ klare Fälle, beispielsweise mit Temporallappenepilepsien auf dem Boden einer Hippokampussklerose diagnostiziert und operiert wurden, stellen sich in den letzten Jahren zunehmend komplexere Patienten vor. Hierunter sind unter anderem Patienten mit ausgedehnten, multifokalen MRT-Läsionen, bei denen geprüft werden muss, von welcher Läsion letztendlich die Anfälle ausgehen. Die größte Gruppe sind derzeit Patienten mit unauffälliger konventioneller MRT-Bildgebung. Diese „MRT-negativen“ fokalen Epilepsien sind eine Herausforderung für die prächirurgische Diagnostik, da eine klare Zielstruktur fehlt (So and Lee, 2014). In diesen Fällen kommt in der Regel ein weites Spektrum komplementärer bildgebender Verfahren zum Einsatz, um Hinweise auf die Lokalisation des Anfallsursprunges zu gewinnen. Diese zunehmende Komplexität der Fälle hat in den letzten Jahren, trotz gestiegener Zahlen an prächirurgischen Untersuchungen, zu einer sinkenden Rate epilepsiechirurgischer Eingriffe geführt, da es nicht immer gelingt, die epileptogene Region zweifelsfrei zu bestimmen (Cloppenborg et al., 2016). Ziel einer modernen prächirurgischen Diagnostik muss es daher sein, durch Kombination komplementärer diagnostischer Methoden auch bei diesen Patienten eine operative Behandlung zu ermöglichen. 
Im Folgenden werden die bildgebenden Methoden, die im Rahmen der prächirurgischen Evaluation zum Einsatz kommen, besprochen, deren Stärken und Schwächen aufgezeigt und schließlich der Zusammenhang zwischen den verschiedenen Methoden erläutert.

\subsection{Magnet-Resonanz-Tomograhie (MRT)}

Verschiedene strukturelle Ursachen einer fokalen Epilepsie stellen sich in der konventionellen MRTUntersuchung des Gehirns gut dar, beispielsweise Tumoren, posttraumatische oder postentzündliche Veränderungen, Blutungen oder Gefäßmalformationen.

Das Fehlen einer in der MRT sichtbaren Läsion ist jedoch nicht ungewöhnlich, da viele typisch epileptogene Pathologien sehr diskret sein können (Woermann and Vollmar, 2009). Fokale kortikale Dysplasien etwa können durch eine Unschärfe der Mark-Rinden-Differenzierung oder durch eine schmale, streifige Hyperintesität in T2 -gewichteten Sequenzen auffallen, die in einer Routine Schädel-MRT-Untersuchung oft nicht abgrenzbar sind. Auch die häufige Hippokampussklerose wird in einer nicht-optimalen MRT-Untersuchung initial oft übersehen. Wiederholte MRT-Untersuchungen mit einem speziellen Epilepsie-Protokoll mit hochauflösenden Schichten über der klinisch vermuteten Anfallsursprungsregion können hier den diagnostischen Gewinn deutlich erhöhen (Von Oertzen et al., 2002). Hierbei wurde gezeigt, dass sowohl die spezifisch optimierte Untersuchungstechnik, als auch die Erfahrung des befundenden Radiologen eine wesentliche Rolle spielen.

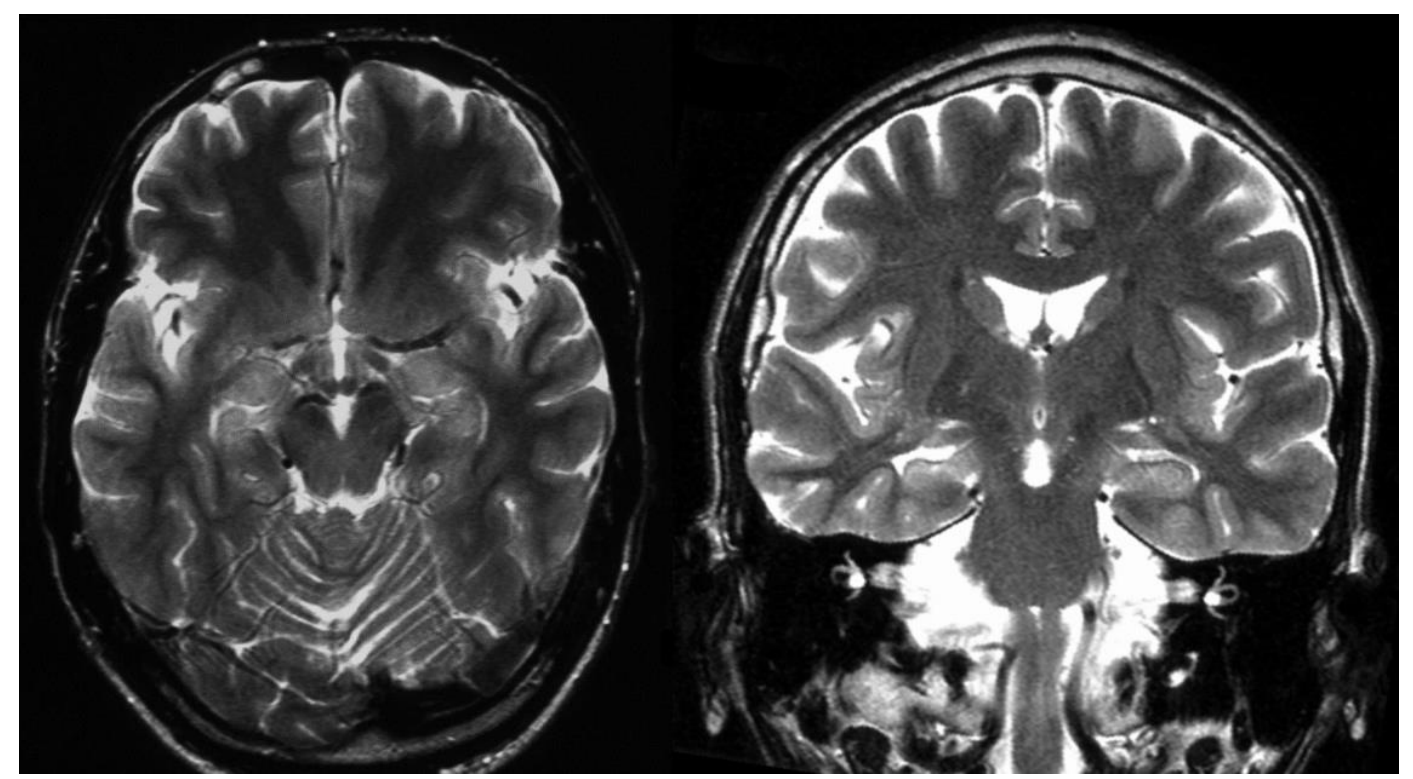

Abbildung 1: T2-gewichtete MRT bei einem Patienten mit rechtsseitiger Hippocampussklerose. Während das linke Bild aus einem Routine-MRT-Protokoll keinen eindeutigen Befund zeigt, stellt sich im rechten, coronaren Bild aus einem speziell optimierten Epilepsie-Protokoll eine deutliche Volumenreduktion und Hyperintensität des rechten Hippocampus dar.

Bevor ein Patient als „MRT-negativ“ klassifiziert wird, muss daher geprüft werden, ob die MRT Untersuchung mit adäquater Untersuchungstechnik in guter, artefaktfreier Bildqualität durchgeführt wurde und von einem erfahrenen Befunder beurteilt wurde.

Doch auch eine optimaler MRT-Untersuchung bleibt bei etwa einem Drittel der Patienten mit fokaler Epilepsie unauffällig. Diese Patientengruppe mit wirklich „MRT-negativer" oder „kryptogener", fokaler Epilepsie stellt eine besondere Herausforderung dar und hier kommen zusätzliche, komplementäre bildgebende Verfahren zum Einsatz (Bernasconi et al., 2011), die unterschiedliche Aspekte des epileptogenen Netzwerkes abbilden. 


\subsection{Positronen-Emissions-Tomographie (PET)}

Die PET erlaubt durch verschiedene Tracer, verschiedene funktionelle oder metabolische Untersuchungen des Gehirns. Am besten etabliert ist die Untersuchung mit Flour-18-markierter Glucose (Flour-18-Deoxy-Glucose, FDG) als Tracer, die es erlaubt, den zerebralen Glucosestoffwechsel darzustellen. Da epileptogenes Gewebe, wie oben erwähnt, meist keine reguläre physiologische Funktion mehr hat, fällt es interiktal, also zwischen den Anfällen, oft durch einen reduzierten Glucosestoffwechsel auf, der diesen Funktionsverlust reflektiert.

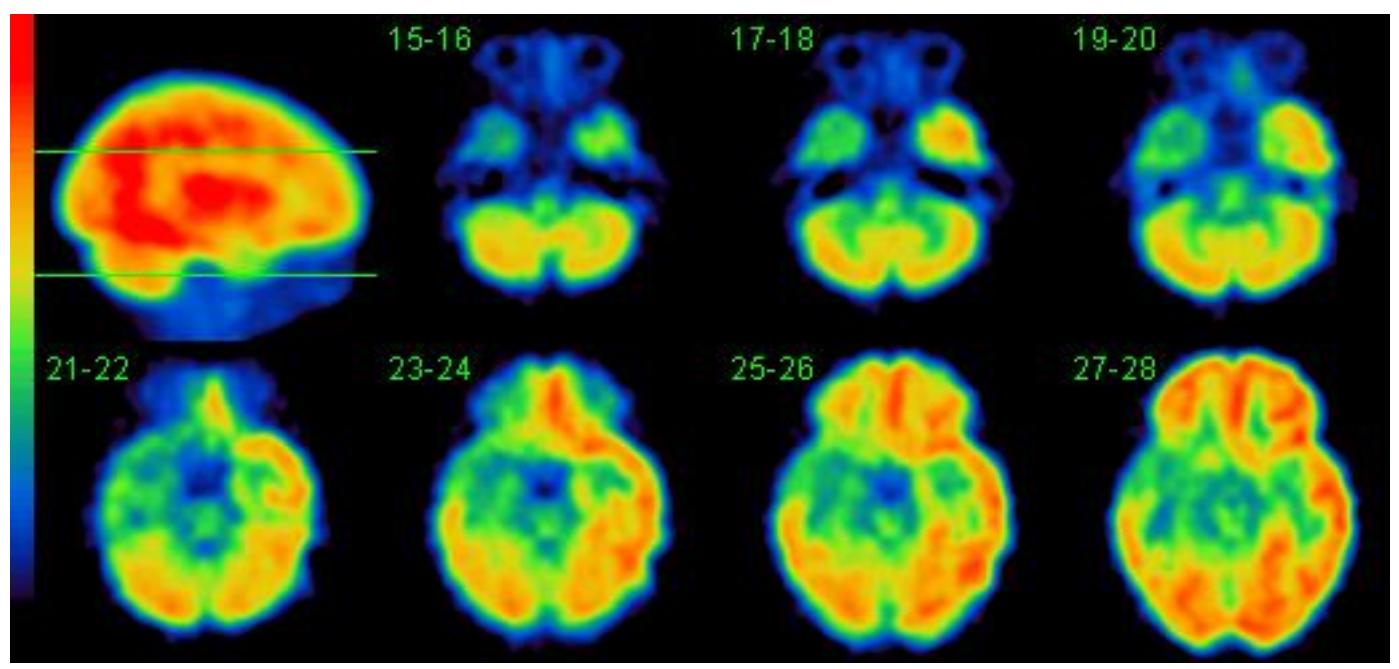

Abbildung 2: Beispiel einer FDG-PET Untersuchung, die einen deutlichen rechts temporalen Hypometabolismus zeigt.

Dieser Hypometabolismus ist meist korrekt lateralisiert, die genaue lokalisatorische Bedeutung ist jedoch gering und muss im Kontext der übrigen klinischen Daten interpretiert werden, da sich oft ausgedehnte, sekundäre Veränderungen in der gleichen Hemisphäre zeigen, zum Beispiel ein zusätzlicher, temporaler Hypo-metabolismus bei Frontallappenepilepsie (Hartl et al., 2016).

\subsection{Single-Photon-Emissions-Computer-Tomographie (SPECT)}

Ein anderes Prinzip liegt der iktalen SPECT-Untersuchung zugrunde: Hier wird unter stationären Bedingungen im Rahmen einer Video-EEG-Monitoring Untersuchung während eines epileptischen Anfalls ein Perfusions-Tracer injiziert, der den aktuellen regionalen Blutfluss zum Zeitpunkt der Tracer-Injektion darstellt. Da ein epileptischer Anfall ein hochaktives Geschehen mit hohem Substratverbrauch ist, zeigt diese Untersuchung eine regionale Perfusionssteigerung im Bereich des Anfallsursprunges (Vollmar and la Fougère, 2012). Der genaue Injektionszeitpunkt ist hierbei entscheidend, da die Untersuchung natürlich auch bereits sekundäre Ausbreitungseffekte zeigen kann. Eine Interpretation der Perfusions-Daten ist daher nur im Kontext der klinischen Information aus EEG und Anfallssemiologie zum Zeitpunkt der Injektion möglich. Die iktale SPECT-Untersuchung hat einen besonders hohen diagnostischen Stellenwert bei MRT-negativen Patienten, bei Patienten mit nicht lokalisiertem oder artefaktverdecktem EEG, sowie bei voroperierten Patienten, bei denen EEG-Befunde wegen veränderter elektrischer Leitfähigkeit durch die Resektion und Knochenlücken schwer zu lokalisieren ist (Bilgin et al., 2008). Nach erfolgreicher iktaler SPECT Untersuchung während eines Anfalles kann ergänzend eine interiktale Untersuchung durchgeführt werden, in der sich die Anfalls-spezifische Perfusionssteigerung nicht zeigt. Durch Subtraktion dieser beiden Untersuchungen kann der Kontrast und damit die Sensitivität und Spezifität der Untersuchung gesteigert werden. 


\subsection{Funktionelle MRT (fMRT)}

Die funktionelle MRT (fMRT) ist eine spezielle MRT Technik, die über eine Veränderung des relativen Anteils von oxygeniertem Hämoglobin Perfusionsveränderungen mit einer hohen zeitlichen Auflösung darstellen kann. Die kontinuierliche Messung während abwechselnder Phasen von Ruhe und Aktivität ermöglicht die Identifikation von Hirnarealen, die unter bestimmten Bedingungen aktiviert werden und erlaubt damit Rückschlüsse auf deren Beteiligung an entsprechenden funktionellen Systemen.

Mit am besten etabliert ist die fMRT zur Lokalisation des primär motorischen Kortex, z.B. durch eine Aktivierung mittels Finger-Tapping. Abbildung 3 zeigt die Lokalisation der Perfusionssteigerung während repetitiver Daumenbewegung, um vor einer geplanten Resektion des unteren Gyrus präzentralis besser abschätzen zu können, wie weit eine Resektion sicher erfolgen kann, ohne ein motorisches Defizit der Hand zu riskieren. In der FDG-PET-Untersuchung stellte sich der untere Gyrus präzentralis deutlich hypometabol dar. Der Anfallsursprung in dieser Region wurde durch invasive EEG Diagnostik mit stereotaktisch implantierten Tiefenelektroden bestätigt.

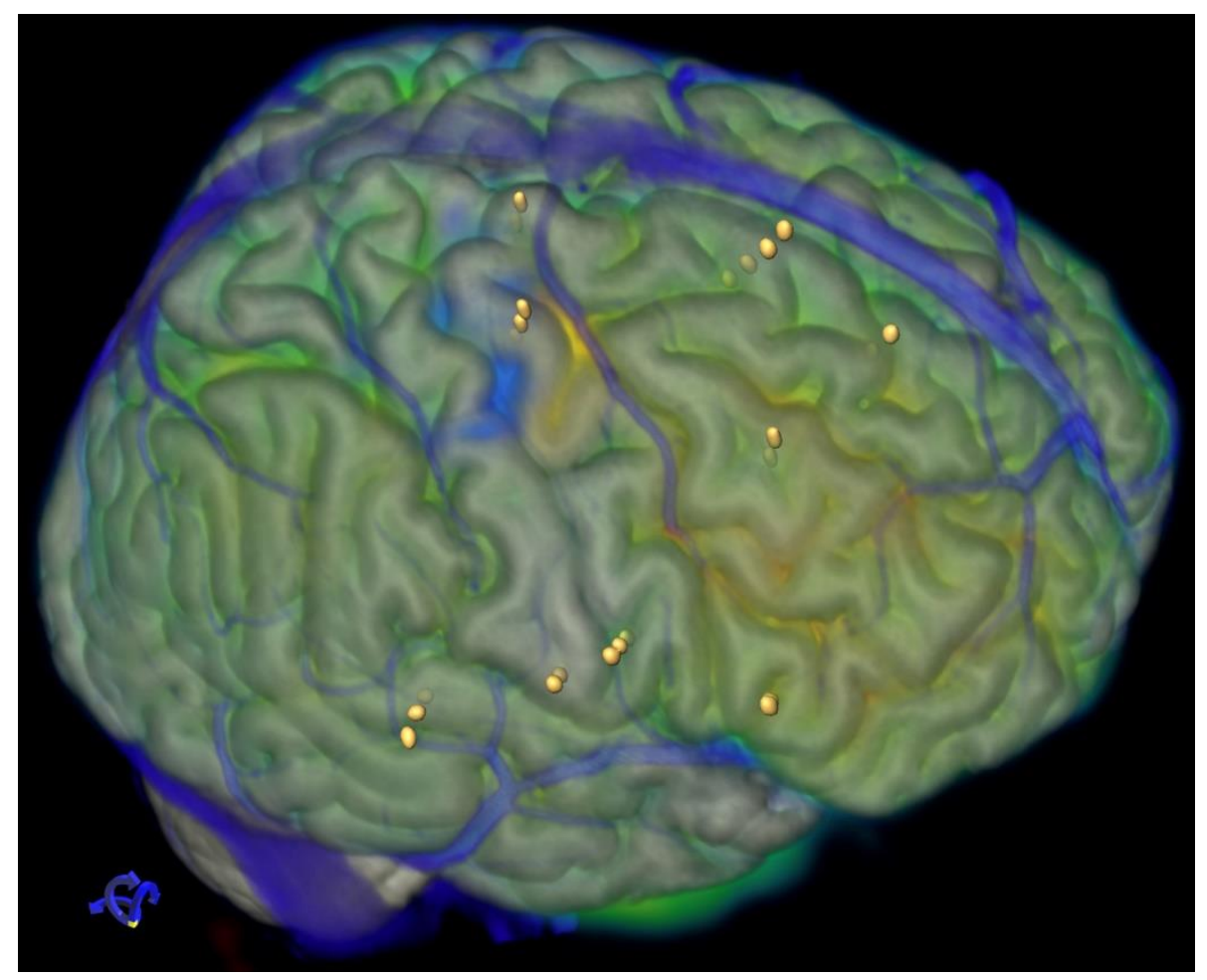

Abbildung 3: kombinierte 3D Rekonstruktion der Kortexoberfläche aus T1-gewichteten MRT Daten. Die flächige Farbcodierung zeigt den Glusosestoffwechel aus einer PET-Untersuchung. Hier stellt sich der untere Teil der Zentralregion hypometabol dar, in dem der Anfallsursprung lokalisiert war. Die blaue Markierung in oberen sulcus centralis zeigt die Perfusionssteigerung während einer fMRT-Untersuchung mit repetitiver Daumen-Bewegung, die deutlich oberhalb des hypometabolen Areals liegt. Direkt davor zeigt die gelbe Markierung die regionale Perfusionssteigerung während eines Anfalls die mittels iktaler SPECT-Untersuchung erhoben wurde. Zum Zeitpunkt der Injektion hatten sich die initialen Kloni der linken Gesichtshälfte schon zu Kloni der linken Hand weiterentwickelt. Die runden gelben Punkte zeigen die Lage stereotaktisch implantierter Tiefenelektroden zur invasiven EEG-Diagnostik. Die blau dargestellten Brückenvenen wurden mittels kontrastverstärkter MRT-Angiographie erfasst.

In vielen Zentren wird die fMRT im Rahmen der prächirurgischen Diagnostik zur Lateralisation der Sprachdominanz eingesetzt (Bonelli et al., 2012). Damit kann beispielsweise vor einem geplanten Eingriff in der linken Hemisphäre eine bilaterale oder evtl. rechts-dominate Sprachrepräsentation nachgewiesen werden. Eine anatomisch exakte Lokalisation von für die Sprache relevanten Arealen ist aufgrund methodischer Limitierungen schwierig. Verschiedene Sprach-Paradigmen führen in der Regel zu unterschiedlichen Aktivierungsmustern, so dass z.B. eine fehlende Aktivierung in einem 
Wortflüssigkeits-Paradigma eine funktionelle Relevanz nicht-aktivierter Areale beim Sprachverständnis nicht ausschließen kann. Zeigt sich dagegen eine Aktivierung, kann die fMRT nicht vorhersagen, ob es bei einer Operation in diesem Bereich wirklich zu einer bleibenden Beeinträchtigung der Sprache käme, oder ob es sich lediglich um akzessorische Anteile des Sprachnetzwerkes handelt, deren Resektion gut kompensiert wird. Bei mehrsprachigen Patienten können verschiedene Sprachen in überlappenden, aber nicht komplett identischen, anatomischen Netzwerken repräsentiert sein (Centeno et al., 2014). Die Sprach-fMRT ist damit ein orientierendes Verfahren und kann die klinische Beurteilung der Sprachfunktion während eines Anfalles (Loesch et al., 2017) ergänzen.

Auch die Identifikation anderer kognitiver Funktionen wie des Arbeitsgedächtnisses (Stretton et al., 2012) oder des Langzeitgedächtnisses (Centeno et al., 2012; Sidhu et al., 2013) sind mittels fMRT möglich und können zu einer besseren Abschätzung von postoperativen kognitiven Veränderungen nach resektiven Eingriffen beitragen.

Allerdings wird die fMRT von vielen zusätzlichen Faktoren beeinflusst, etwa durch unterschiedliche kognitive Strategien bei verschiedenen Patienten, oder durch Medikamenteneinnahme, die teilweise regional spezifische Effekte auf die fMRT Aktivierung hat (Yasuda et al., 2013). Daher muss die fMRT meist durch zusätzliche Verfahren, insbesondere die elektrische Kortexstimulation, ergänzt werden, die aktuell den Goldstandard repräsentieren, um funktionelle Areale sicher zu identifizieren (Guldiken et al., 2015; Borggraefe et al., 2016).

\subsection{Diffusion Tensor Imaging (DTI)}

Das DTI ist eine neuere MRT Methode, die auf der Messung der Diffusionsfähigkeit von Wasser basiert. Durch Messung der Diffusionsfähigkeit in verschiedenen Raumrichtungen lässt sich nicht nur ein Gesamtmaß für die Diffusionsfähigkeit bestimmen, sondern auch, wie stark die Diffusion in einer bestimmten Richtung dominiert. Ein Maß für diese Ausrichtung der Diffusion ist die fraktionelle Anisotropie (FA), deren Wert bei völlig ungerichteter Diffusion bei 0 liegt und im Falle von ausschließlich auf eine einzige Raumrichtung beschränkter Diffusion ein Maximum von 1 erreicht. Die höchsten FA Werte zeigen sich in Regionen mit besonders dicht gepackten axonalen Bündeln, die bevorzugt in einer Richtung verlaufen, wie im dem Corpus Callosum. Häufig werden FA Werte als Maß für die strukturelle Integrität der weißen Substanz interpretiert, die bei verschiedensten neurologischen Erkrankungen vermindert sein kann. Schließlich kann aus der Diffusionsfähigkeit in verschiedenen Richtungen auch der Vektor der Hauptdiffusionsrichtung berechnet werden. In der weißen Substanz des menschlichen Gehirnes korreliert dieser Vektor gut mit der Verlaufsrichtung der axonalen Faserbündel. Das Verfahren, bei dem man von Bildpunkt zu Bildpunkt dem Verlauf dieser Vektoren folgt, wird daher auch vereinfachend als "fiber tracking" bezeichnet und kann die bekannten anatomischen Verbindungsbahnen des Gehirns darstellen. 


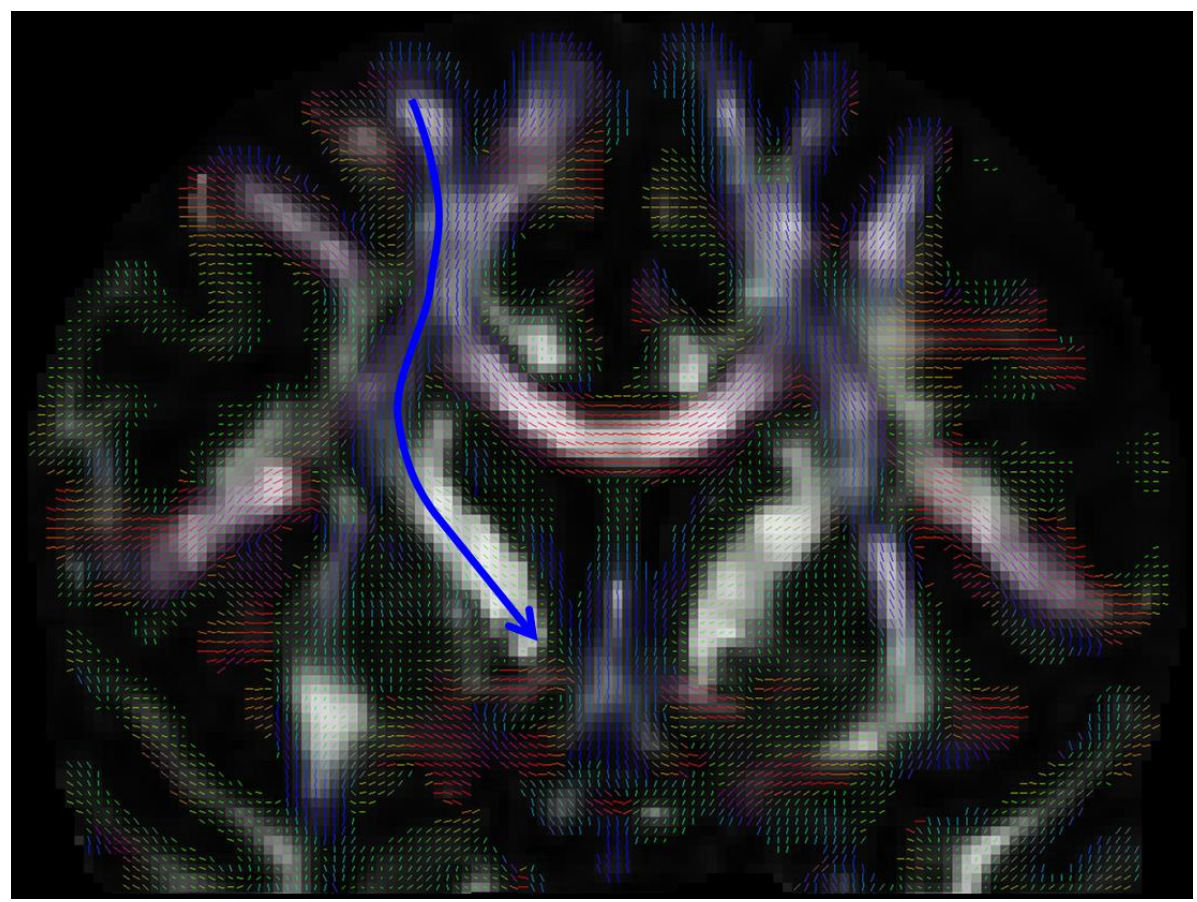

Abbildung 4: DTI: Das Hintergrundbild zeigt eine FA Werte, die im Bereich dicht gepackter, streng ausgerichteter Faserbündel am höchsten sind. Farbcodiert überlagert sind die Vektoren der Hauptdiffusionsrichtung in jedem Bildpunkt dargestellt. Die blaue Linie illustriert den Tracking prozess, bei dem man von einem Startpunkt aus der Kette von Diffusionsvektoren folgt.

Im Unterschied zur fMRT ist die DTI als strukturelle Bildgebungsmethode sehr robust und gut reproduzierbar (Vollmar et al., 2010). Trotzdem ist es wichtig, sich der Einschränkungen der Methode bewusst zu sein, insbesondere der Tatsache, dass hierbei natürlich keine neuroanatomischen Fasern dargestellt werden, sondern Vektoren von Wasser-Diffusion mit einer ca. tausendfach gröberen Auflösung.

Im Rahmen der prächirurgischen Epilepsiediagnostik kann die DTI mit verschiedenen Fragestellungen zum Einsatz kommen (Vollmar and Diehl, 2011):

Zum einen ist es möglich, durch anatomisch gezieltes Tracking funktionell relevante Verbindungsbahnen darzustellen, die für die Planung von operativen Eingriffen limitierend sein können. Bei geplanten Eingriffen nahe der Zentralregion ist zum Beispiel die Darstellung der absteigenden, motorischen Bahnen im kortikospinalen Tract relevant, um diese zu schonen (Kovac et al., 2010). Der kortikospinale Tract ist eines der prominentesten Faserbündel der weißen Substanz und seine Darstellung gelingt selbst mit relativ einfachen DTI Daten in niedriger Qualität.

Die erste regelmäßige Anwendung bei epilepsiechirurgischen Eingriffen fand das Tracking funktionell relevanter Bahnen bei Patienten mit Temporallappenepilepsie, bei denen eine antero-mesiale Temporallappenteilresektion durchgeführt wurde. Die Sehstrahlung vom corpus geniculacum laterale zum primären visuellen Kortex ist im Laufe der Entwicklungsgeschichte mit dem wachsenden Temporallappen des menschlichen Gehirns weit nach vorne in den Temporallappen verlagert worden. Hier verläuft sie nun im Dach des Seitenventrikel-Unterhornes und ist bei einer Temporallappenresektion gefährdet. Hier zeigte sich, dass die präoperative Darstellung und Vermessung der Sehstrahlung mittels DTI das Auftreten und das Ausmaß von postoperativen Gesichtsfelddefekten reduzieren kann (Yogarajah et al., 2009). 


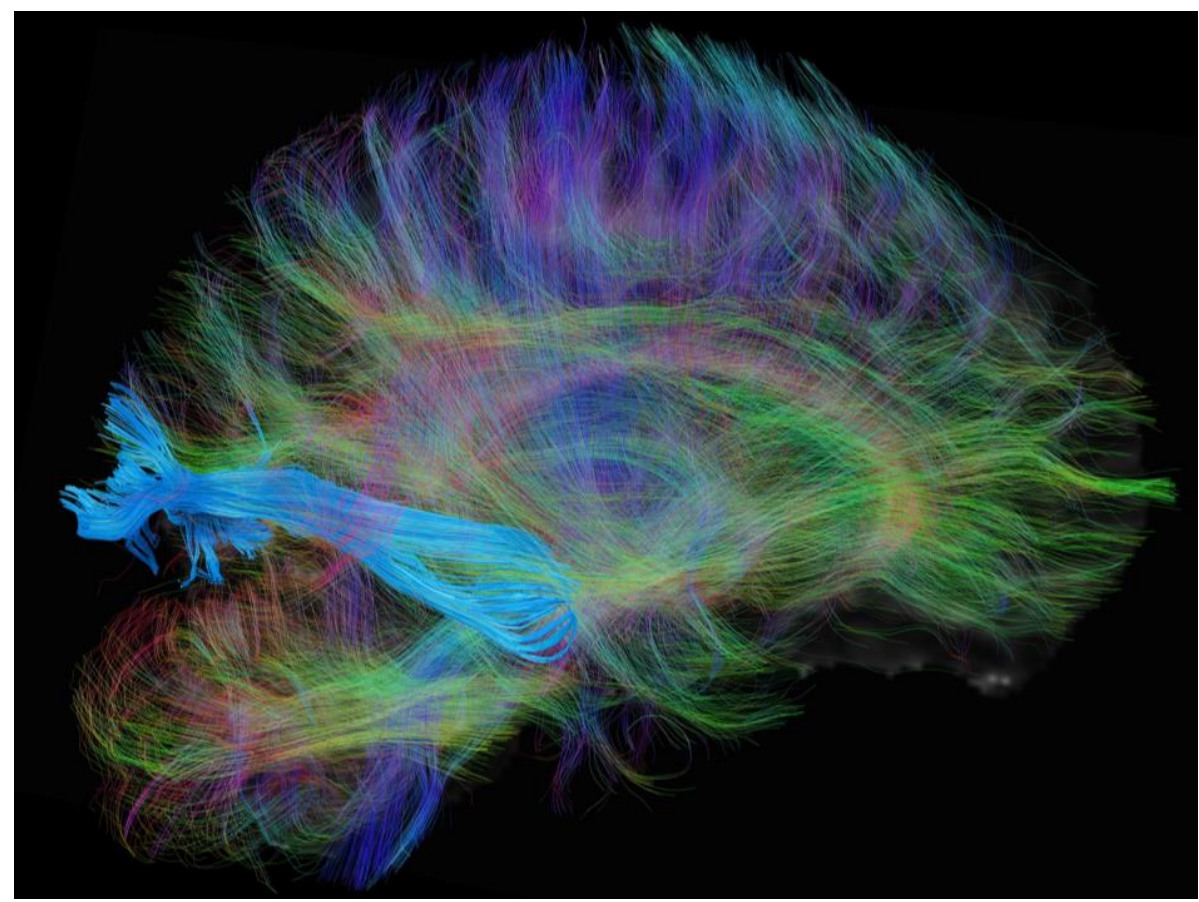

Abbildung 5: DTI Fiber Tracking: Diese laterale Ansicht auf die rechte Seite zeigt in blau die Verbindungen der Sehstrahlung, vom corpus geniculacum laterale zum occipitalen visuellen Kortex. In Hintergrund sind für eine anatomische Orientierung die Verbindungen einer sagittalen Ebene durch den Temporallappen und ein transparentes FA-Bild zu sehen.

Aus DTI Daten kann eine Vielzahl von Parametern rekonstruiert werden, die Hinweise auf verschiedene mikrostrukturelle Eigenschaften des Gewebes liefern. Bei Epilepsie Patienten mit unauffälliger konventioneller MRT-Bildgebung stellt die DTI somit potentiell eine zusätzliche, unabhängige Methode dar, die Hinweise auf die Lokalisation des gesuchten epileptogenen Areals liefern kann.

Erste visuelle Analysen nach „whole brain tracking" haben hier gezeigt, dass sich in den klinisch vermuteten Regionen oft eine verminderte Dichte von rekonstruierten Faserbündeln zeigt (Goc et al., 2014). Während die tief gelegenen, großen, prominenten Faserbündel kaum Veränderungen zeigten, waren die peripheren U-Fasern, die benachbarte Gyri miteinander verbinden, besonders oft betroffen. Dies erschien plausibel, da histologische Untersuchungen bei MRT-negativen EpilepsiePatienten besonders häufig fokale kortikale Dysplasien (FCD) als ursächliche Pathologie zeigten. Diese FCD liegen typischerweise an der Grenze zwischen Kortex und weißer Substanz, also genau dort, wo die direkt subkortikal liegenden U-Fasern verlaufen. Offenbar ist der DTI Tracking Prozess sensitiv genug, um auch von diskreten Veränderungen in diesem Bereich gestört zu werden, was letztendlich zu einer verminderten Dichte der rekonstruierten Fasern führt. 


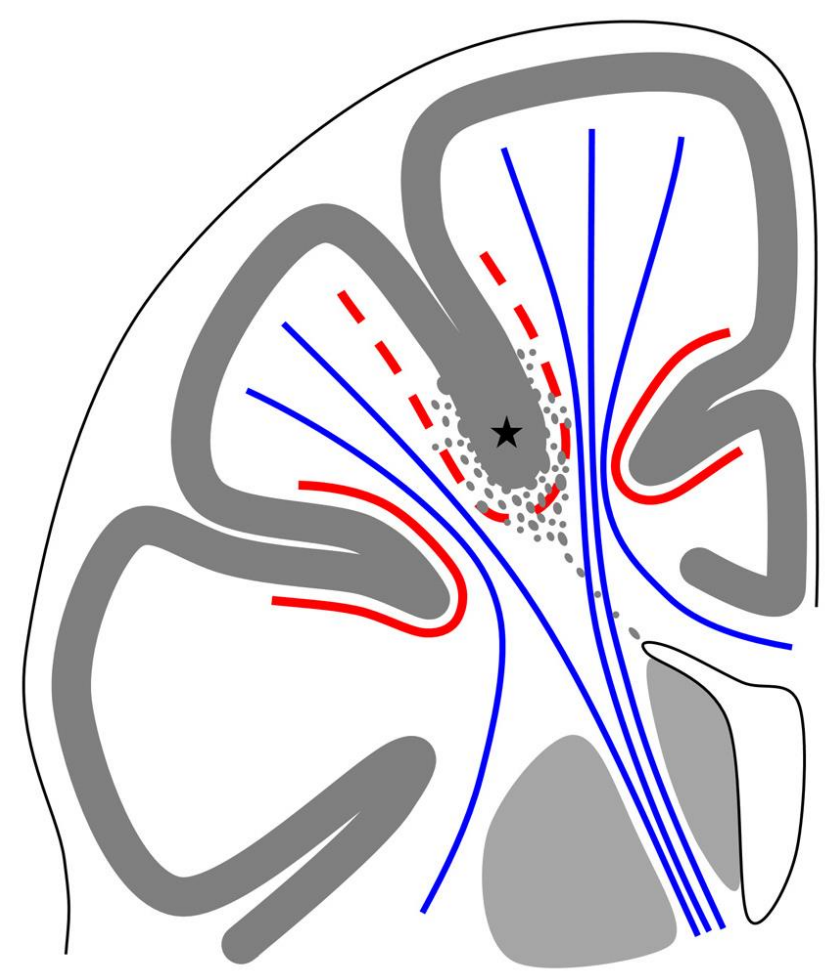

Abbildung 6: Fokale cortikale Dysplasien liegen meist in der Tiefe eines Sulcus an der Mark-Rinden-Grenze $\left({ }^{*}\right)$ die blau dargestellten langen Projektionsbahnen sind hiervon zu weit entfernt um betroffen zu sein. Die subcortikal verlaufenen UFasern (rot), die benachbarte Gyri miteinander verbinden liegen dagegen genau in diesem Bereich. Hier scheinen einige ektope Neurone im subkortikalen Marklager den Tracking Prozess so zu stören, dass sich eine regionale Verminderung der U-Faser Dichte zeigt.

Hierauf basierend wurde in den letzten Jahren eine Methode entwickelt, die selektiv die regionale Dichte an U-Fasern quantifiziert und durch Vergleich mit einer gesunden Kontrollpopulation statistisch signifikante Reduktionen identifizieren kann (Vollmar et al., 2016) Korrelationen mit den klinischen Daten zeigen hier eine hohe Rate von auffälligen Reduktionen der U-Faser-Dichte in der klinisch identifizierten Anfallsursprungszone. Diese zusätzliche Information wird mittlerweile regelmäßig genutzt, um die Implantation von intrakraniellen Elektroden zur invasiven EEG-Diagnostik zu planen.

Als proof-of-concept wurde die gleiche DTI Untersuchung bei einem Patienten mit hypoxischer Hirnschädigung durchgeführt. Hier zeigte sich eine spezifische, regionale Reduktion subkortikaler UFasern rechts parietal, die zu einer verminderten Inhibition mit kortikaler Übererregbarkeit, Riesenpotentialen und epileptischen Anfällen führte (Feddersen et al., 2015).

Neben dieser konkreten klinischen Anwendung bei einzelnen Patienten kann die DTI für zahlreiche wissenschaftliche Fragestellungen eingesetzt werden, zum Beispiel um Veränderungen nach epilepsiechirurgischen Eingriffen zu untersuchen. Hier zeigten sich nach Resektionen des Temporallappens ausgedehnte Effekte auf extratemporale Regionen. Neben den zu erwartenden Verminderungen der FA Werte in angrenzenden Regionen zeigten sich auch FA-Erhöhungen im ventro-medialen Sprachnetzwerk, die mit einer guten postoperativen Sprachfunktion korrelierten und somit offenbar eine erfolgreiche strukturelle Reorganisation nach dem operativen Eingriff reflektieren (Yogarajah et al., 2010).

Auch außerhalb der Epilepsien kann eine quantitative Analyse von DTI Daten pathologische Veränderungen jenseits einer unauffälligen konventionellen MRT identifizieren und sogar als Verlaufsparameter zum Therapie-Monitoring dienen (Catarino et al., 2017). 


\subsection{Lokalisationskonzept bei fokaler Epilepsie}

Die vorangegangenen Abschnitte haben gezeigt, dass verschiedene bildgebende Methoden zum Teil sehr unterschiedliche Aspekte eines komplexen, epileptogenen Netzwerkes reflektieren. In seltenen, einfachen Fällen fallen all diese Aspekte räumlich sehr eng zusammen, d.h. jede diagnostische Methode zeigt die pathologischen Befunden an der gleichen Stelle, insbesondere zeigt die MRT dort eine strukturelle Läsion. Nur in diesen Fällen kann man wirklich von einem „Focus“ sprechen.

Bei dem selektierten Patientengut eines Epilepsie-Referenzzentrums ist dies jedoch nur selten der Fall. Nach vielen Jahren chronischer Epilepsie und Reorganisation sind die Befunde oft teilweise diskrepant und reflektieren oft ausgedehnte epileptogene Netzwerke und sekundäre Veränderungen. Zudem kann jede einzelne Methode in der Diagnostik versagen und unauffällige Befunde zeigen, oder sogar durch falsch positive Befunde zusätzliche Verunsicherung schaffen. Aufgabe der prächirurgischen Evaluation ist es daher nicht nur, all diese Befunde zu erheben, sondern vor allem eine konklusive Interpretation sämtlicher Befunde im klinischen Kontext durchzuführen. Dies führt in der Regel zu einem sehr individuellen Mischbild aus diagnostischen Methoden, die einen sehr unterschiedlichen Beitrag zum Gesamtbild beisteuern. Die MRT kann eine einzelne, hochspezifische Läsion zeigen, oder multiple Läsionen, von denen eine den Anfallsursprung reflektiert, oder ausgedehnte, unspezifische Veränderungen, oder sie kann ganz unauffällig sein. Ebenso kann eine PET-Untersuchung einen eindeutig pathologischen Befund in einer umschriebenen Region zeigen, oder aber einen ausgedehnten Hypometabolismus einer gesamten Hemisphäre, oder auch einen unauffälligen Befund ergeben. Das gleiche gilt sinngemäß für jede weitere diagnostische Methode wie interiktales und iktales EEG, Anfallssemiologie, SPECT oder DTI.

Durch welche Kombination aus Methoden auch immer, steht am Ende der prächirurgischen Evaluation die Definition der vermuteten epileptogenen Zone, also des Areals, das für die Entstehung der Anfälle verantwortlich ist und das reseziert werden muss, um die Anfälle zu kontrollieren. 


\subsection{Bildverarbeitung und multimodale Bildgebung}

Diese konklusive Interpretation multipler, diagnostischer Methoden kann nicht anhand schriftlicher Befunde durchgeführt werden. Gerade die Vielzahl der bildgebenden Informationen, die im Rahmen der prächirurgischen Epilepsiediagnostik erhoben werden, macht es nötig, diese sinnvoll aufzubereiten und zu kombinieren. Diese multimodale Bildgebung basiert auf zwei wesentlichen Elementen: der Bildfusion und der kombinierten 3D-Visualisierung. Die Bildfusion bringt Bilddaten verschiedener Modalitäten räumlich zur Deckung und erlaubt damit eine anatomisch exakte Korrelation von verschiedenen Befunden (Pfluger et al., 2000). Insbesondere für die Planung von chirurgischen Eingriffen ist es sinnvoll, die Daten im Kontext der individuellen, gyralen Anatomie eines Patienten dreidimensional darzustellen. Da diese am besten aus einem hochauflösenden T1gewichteten MRT Datensatz rekonstruiert werden kann, stellt dieser in der Regel den Basis-Datensatz dar, auf den alle anderen Modalitäten koregistriert werden.

Die multimodalen Bildgebung für die Epilepsiediagnostik wurde vor vielen Jahren in München etabliert und im Verlauf der Jahre stetig weiterentwickelt (Vollmar et al., 2008). Die zusätzliche Einbindung neuer bildgebender Verfahren wie der fRMT oder DTI erweitert das Spektrum der multimodalen Bildgebung und technische Weiterentwicklungen im Bereich der Bildakquisition und verarbeitung erlauben eine immer bessere Bildqualität der 3D-Rekonstruktionen (Vollmar, 2012). Die Fusion mit einem CT-Datensatz, der intrakraniell implantierte Elektroden darstellt, erlaubt schließlich die direkte Korrelation von neurophysiologischen und bildgebenden Befunden in einem gemeinsamen 3D-Bild (Winkler et al., 2000) und damit eine vollständig konklusive Beurteilung sämtlicher vorliegender Daten. Diese Kombination aller verfügbaren Informationen erleichtert die Interpretation einzelner Befunde und erlaubt eine zuverlässigere Lokalisation sowohl der zu resezierenden, epileptogenen Areale, als auch der zu schonenden, eloquenten Areale. Unter Berücksichtigung der individuellen, anatomischen Verhältnisse ist so eine hochindividualisierte, „maßgeschneiderte“ Planung des chirurgischen Eingriffes möglich (Vollmar et al., 2008) (Abb. 7).

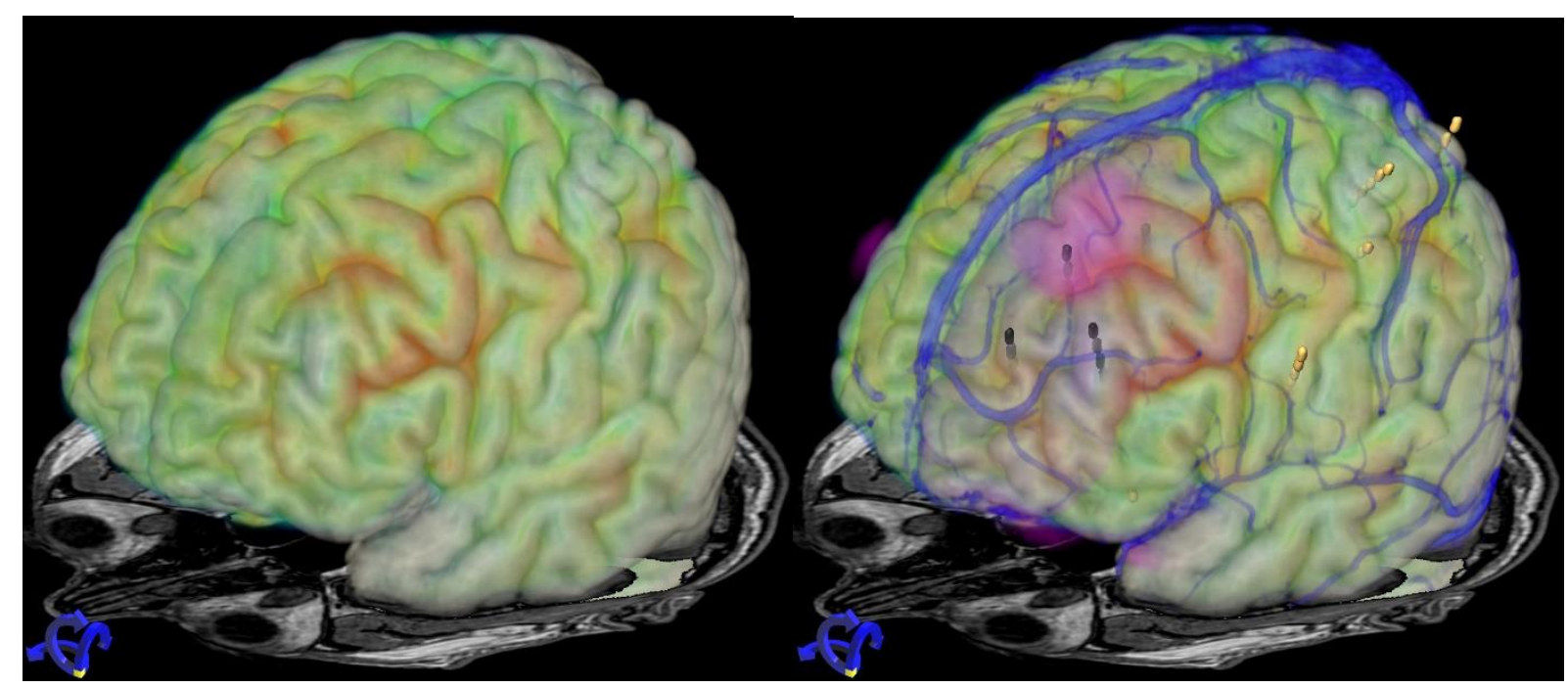

Abbildung 7: Multimodale 3D Rekonstruktion eines Patienten mit MRT-negativer Frontallappenepilepsie links. Wie in Abb. 3 ist die Kortexoberfläche aus einer T1-gewichteten MRT rekonstruiert, die Farbcodierung zeigt den Glucosestoffwechsel, der eine kokale Verminderung im vorderen Anteil des Gyrus frontalis medius zeigte. Das rechte Bild zeigt zusätzlich in pink eine Region mit signifikant verminderter U-Faser-Dichte in der quantitativen DTI Auswertung. Die schwarz markierten Elektrodenkontakte zeigten einen ausgedehnten Anfallsursprung. Die lila-orange Kontur reflektiert das geplante Resektionsvolumen des linken Gyrus frontalis superior und medius.

Eine Implementation dieses Ansatzes in London hat zur Entwicklung einer spezifischen Planungssoftware für die Implantation von Tiefenelektroden geführt (Rodionov et al., 2013). 


\subsection{Intraoperative Anwendung der Bildgebung}

Letztendlich läuft eine erfolgreiche prächirurgische Evaluation auf einen epilepsiechirurgischen Eingriff hinaus, und die erhobenen Befunde müssen während der Resektion im OP Saal konkret umgesetzt werden. Jede Unsicherheit bezüglich der exakten Lokalisation von Befunden würde zu einer kleineren, defensiveren Resektion führen, die zwar weniger Risiken birgt, aber auch geringere Chancen auf Anfallsfreiheit bietet

Die 3D Darstellung der individuellen gyraler Anatomie zeigt oft charakteristische Landmarken, die auch im OP-Feld identifiziert werden können und der Orientierung dienen. Die Integration von Bildern einer MRT-Angiographie-Untersuchung in die multimodale Bildgebung erlaubt zusätzlich die Darstellung oberflächlicher Blutgefäße, insbesondere der Venen, als weitere Landmarken (Vollmar et al., 2008). Die Gefäße sind in der neurochirurgischen Praxis meist leichter zu identifizieren als Details der gyralen Anatomie, die oft von genau diesen Gefäßen verdeckt sein können. Im Gegensatz zu den üblichen neurochirurgischen Navigationssystemen, die auf einem präoperativ angefertigten MRT basieren, ist die Orientierung anhand der multimodalen 3D Rekonstruktionen unempfindlich gegenüber einem sogenannten brain-shift, also der Verschiebung von Hirngeweben nach einer großen Kraniotomie. Die Lokalisation relevanter Befunde in Relation zu charakteristischen GefäßLandmarken bleibt immer gleich, auch wenn die gesamte Kortexoberfläche nach Dura-Eröffnung und Liquorverlust um einen Zentimeter eingesunken ist (Abb. 8).

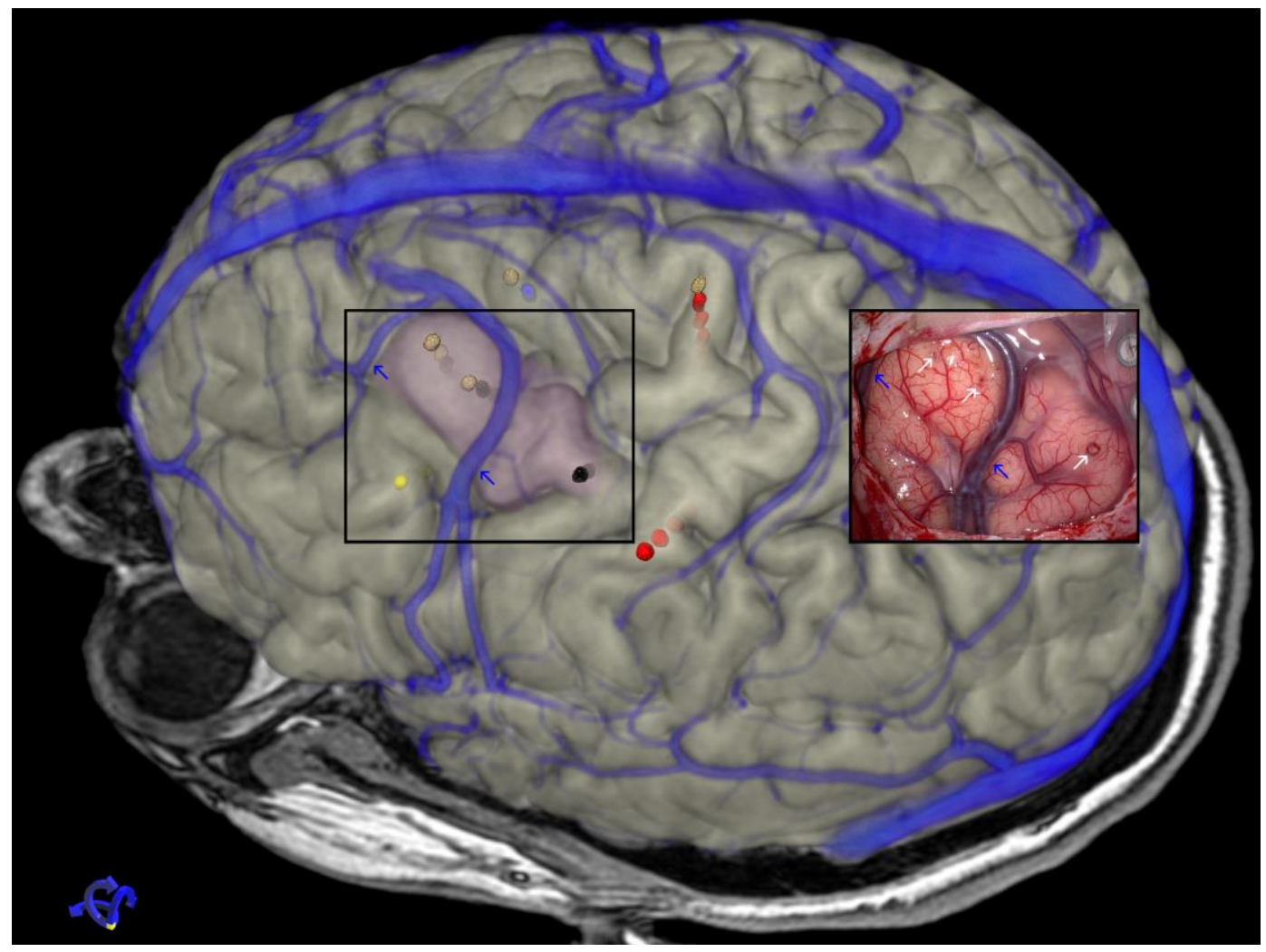

Abbildung 8: Vergleich der multimodalen 3D Rekonstruktion mit dem korrespondierenden OP Feld. Die oberflächlichen Venen (blaue Pfeile) sind verlässliche anatomische Landmarken, an denen man sich intraoperativ gut orientieren kann. Auch die Struktur der gyralen Anatomie ist gut zwischen dem 3D Bild und dem OP-Situs vergleichbar. Die früheren Eintrittsstellen von Tiefenelektroden sind als winzige, punktförmige Einblutungen (weiße Pfeile) erkennbar. Die farbcodierten Elektrodenkontakte zeigen in schwarz den Anfallsursprung sowie die Effekte der extraoperativen elektrischen Stimulation, die Sprache (gelb), Motorik (rot) und negativ motorische Antworten (blau) identifizieren konnte. Da das lila markierte Resektionsvolumen in den Gyrus präzentralis reichte, wurde eine intraoperative elektrische Stimulation des Kortex ergänzt um die maximal mögliche Resektion ohne motorisches Defizit zu erreichen. 
Daher werden die multimodalen 3D Rekonstruktionen seit vielen Jahren regelmäßig zur intraoperativen Orientierung eingesetzt. Während dies früher mit Papierausdrucken oder statischen Bildern auf dem Laptop umgesetzt wurde, ermöglicht die aktuelle moderne OP-Infrastruktur und ausreichend leistungsfähige Laptops heute eine dynamische Anwendung, bei der die gerade aktuelle Ansicht des OP-Feldes in den multimodalen Datensätzen in Echtzeit im OP nachgestellt werden kann und direkt am OP-Tisch auf einem Bildschirm verfügbar ist (Abb.9). Einzelne Modalitäten können hierbei dynamisch ein- und ausgeblendet werden, die Transparenz jeder Komponente kann interaktiv angepasst werden um jederzeit die gerade relevanten Aspekte der prächirurgischen Diagnostik zu visualisieren. Mittlerweile wird regelmäßig auch das geplanten Resektionsvolumen als 3D Objekt innerhalb der multimodalen Daten definiert und kann zu jedem Zeitpunkt der Resektion eingeblendet werden.

Diese innovative Anwendung der multimodalen Bildgebung erlaubt eine zuverlässige Übertragung präoperativer Befunde in das OP-Feld und bietet damit mehr Sicherheit bei komplexen Eingriffen. Insbesondere bei Resektionen in der Nähe von eloquenten Kortex oder bei MRT-negativen Patienten, wo eine offensichtliche Zielstruktur fehlt, erlaubt diese zusätzliche Sicherheit durch intraoperativ verfügbare multimodale Daten eine vollständige Resektion der geplanten Areale bei gleichzeitig bestmöglicher Schonung eloquenter Regionen.

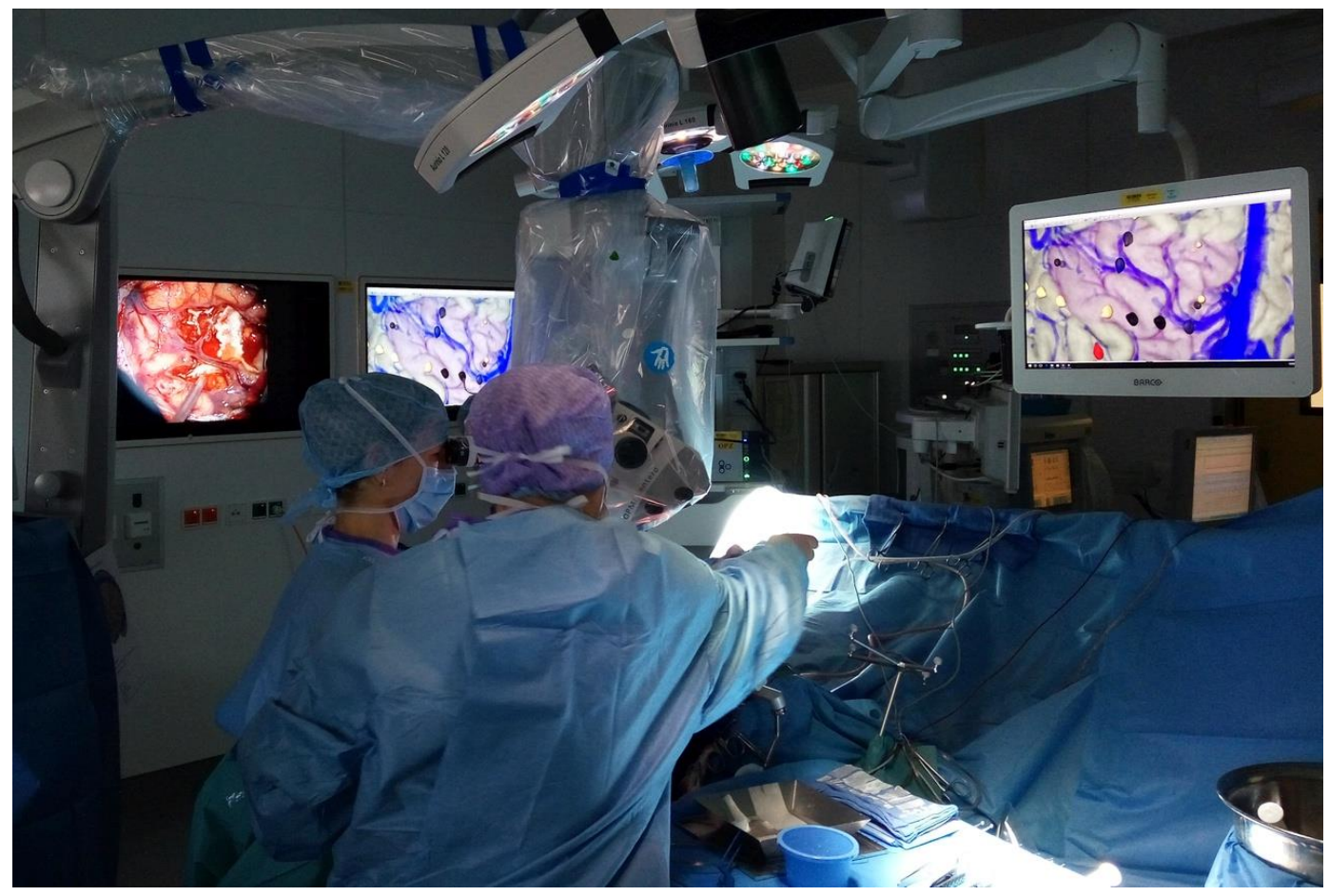

Abbildung 9: intraoperative Anwendung der multimodalen Bildgebung. Die aktuelle Ansicht des OP-Feldes durch das Mikroskop ist auf dem einen Wandmonitor sichtbar. So kann die entsprechende Ansicht in den 3D Daten nachgestellt und am Decken-Monitor des OP-Feldes sichtbar gemacht werden. Die Sichtbarkeit der einzelnen diagnostischen Modalitäten, Transparenzen, Farbschemata etc. können interaktiv der jeweils aktuellen Frage angepasst werden. 


\subsection{Ergebnisse nach epilepsiechirurgischen Eingriffen}

Historisch liegt die Rate von anfallsfreien Patienten nach einem Epilepsiechirurgischen Eingriff bei ca. 60\%. Zwar haben sich die Ergebnisse bei temporalen Resektionen in neueren Studien auf bis zu 80\% verbessert, insbesondere bei extratemporalen Resektionen, oder bei MRT-negativen Patienten berichten jedoch auch neuere Studien Anfallsfreiheitsraten zwischen 15 und 44\% (Ryvlin et al., 2014; West et al., 2016).

Eine aktuelle Auswertung von 70 Patienten unseres Zentrums, mit epilepsiechirurgischen Eingriffen nach invasiver Diagnostik mittels stereotaktisch implantierter Tiefenelektroden zeigte ein hervorragendes Ergebnis mit insgesamt 83\% anfallsfreien Patienten (Thorsteinsdottir et al., 2018) (Abb. 10). Insbesondere gab es in der hier behandelten Serie keine signifikanten Unterschiede zwischen Patienten mit und ohne MRT-Läsion, keine Unterschiede zwischen temporalen und extratemporalen Resektionen und keine Unterschiede zwischen links- und rechtshemisphärischen Resektionen. Auch die Nähe der Resektion zu eloquentem Kortex hatte keine Auswirkung auf das postoperative Ergebniss.

Outcome after SEEG and resection (average follow up 41.7 months)

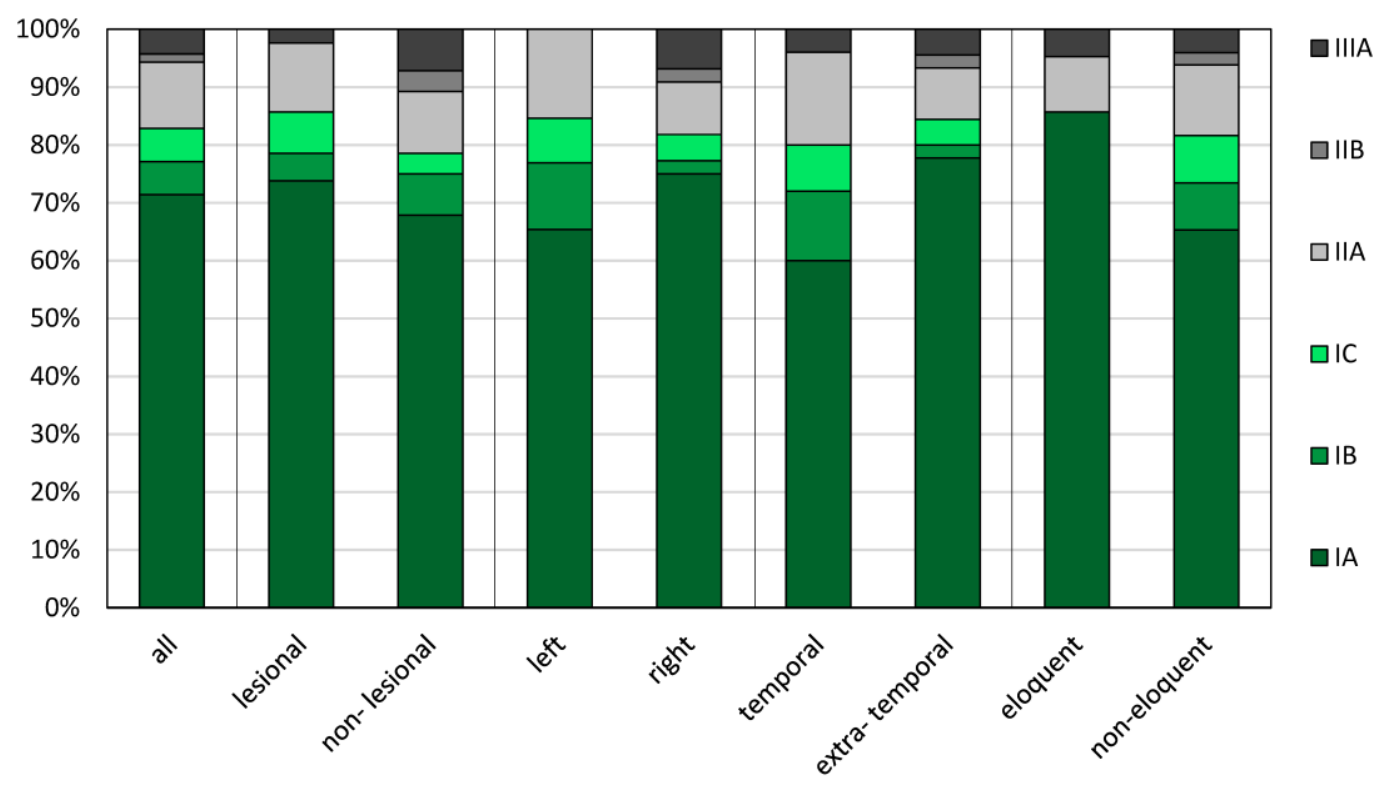

Abbildung 10: Ergebnisse nach epilepsiechirurgischen Eingriffen. Die Details der Klassifikation nach Engel sind in Tabelle 1 aufgeführt. Zusammengefasst gelten Patienten in Class 1, die hier grün dargestellt sind, als „anfallsfrei“. Dieses Ziel wurde bei $83 \%$ der Patienten erreicht.

Tabelle1: Engel Classification of seizure outcome after surgery

Class 1: Free of disabling seizures

1A: Completely seizure-free since surgery

1B: Non-disabling simple partial seizures only since surgery

1C: Some disabling seizures after surgery, but free of disabling seizures for at least 2 years

1D: Generalised convulsion with antiepileptic drug withdrawal only

Class 2: Almost seizure-free (rare disabling seizures)

2A: Initially free of disabling seizures but has rare seizures now

2B: Rare disabling seizures since surgery

2C: More than rare disabling seizures after surgery, but rare seizures for at least 2 years

2D: Nocturnal seizures only 
Class 3: Worthwhile improvement

3A: Worthwhile seizure reduction

3B: Prolonged seizure-free intervals ( $>$ half the follow up period), but not less than 2 years

Class 4: No worthwhile improvement

$4 \mathrm{~A}$ : No significant seizure reduction

4B: No appreciable change

4C: Seizures worse

Diese Ergebnisse stellen einen wesentlichen Unterschied zu bisher publizierten Daten dar, wo in bestimmten Subgruppen teilweise nur 20-40\% Anfallsfreiheit erzielt wurden. Auch der Anteil der zumindest "fast anfallsfreien" Patienten, die nur noch sehr selten Anfälle haben war in unserer Serie hoch. In allen Subgruppen gab es jeweils nur wenige Prozent an Patienten mit einem outcome von $3 \mathrm{~A}$, d.h. zumindest eine nennenswerte Reduktion der Anfallsfrequenz. In der ganzen Serie gab es keinen Patienten der gar nicht von der Resektion profitiert hatte.

Das Ergebnis solch komplexer Therapien stellt immer die Summe vieler Faktoren dar, beginnend mit der Auswahl geeigneter Patienten, der Qualität der einzelnen prächirurgischen Untersuchungen, der Interpretation der Befundkonstellation, der operativen Technik und vieler anderer. Gerade im Vergleich zu anderen Zentren ist die in München etablierte multimodale Bildgebung und intraoperative Navigation jedoch ein herausragender Baustein des Epilepsiechirurgie-Programms. Zumindest ein relevanter Anteil der guten Ergebnisse kann daher sicherlich der Qualität der bildgebenden Diagnostik zugeschrieben werden. Insbesondere die guten Ergebnisse bei MRTnegativen Patienten deuten an, dass das Fehlen einer MRT-Läsion durch eine breit aufgestellte multimodale Bildgebung mit komplementären Methoden gut kompensiert werden kann. Die gleich guten Ergebnisse bei Resektionen in der linken Hemisphäre und angrenzend an eloquenten Kortex zeigen, dass eine große Sicherheit bei der Identifikation eloquenter Areale bestand und in diesen Fällen nicht aus Sorge vor postoperativen Defiziten kleinere, weniger erfolgreiche Resektionen durchgeführt wurden. 


\subsection{Ausblicke - klinische Anwendung}

Die bereits etablierten Techniken zur Bildfusion und 3D Visualisierung werden bereits routinemäßig in der klinischen Versorgung eingesetzt. Potential zum weiteren Ausbau liegt hier z.B. in der Inklusion weiterer diagnostischer Methoden, wie der aktuell neu verfügbaren Flumazenil-PET-Untersuchung, die über die Darstellung von GABA-Rezeptoren einen neuen Baustein zur Identifikation epileptogener Veränderungen darstellt. Auch die präoperative Visualisierung von Befunden kann durch konsequente Verwendung neuer Techniken, wie der stereoskopischen Visualisierung oder der Erstellung individueller, anatomischer Modelle mittels 3D-Druck, für die OP-Planung noch ausgebaut werden. Eine engere Anbindung an neurochirurgische Navigationssysteme könnte die exakte Umsetzung von Ergebnissen der präoperativen Untersuchungen im OP-Saal weiter verbessern.

\subsection{Ausblicke - wissenschaftliche Anwendungen}

Epilepsien werden heute zunehmend als Netzwerk-Erkrankungen verstanden, bei denen es zwar im Falle fokaler Epilepsien eine kritische Region gibt, in der die Anfälle generiert werden, deren klinisches Bild aber zusätzlich von zahlreichen Faktoren beteiligter zerebraler Netzwerke beeinflusst werden (M Centeno et al., 2014). Im Rahmen der prächirurgischen Diagnostik werden verschiedene diagnostische Methoden eingesetzt, die sich für die Analyse solcher Netzwerke eignen: Die routinemäßig durchgeführte Elektroenzephalographie erlaubt über Kohärenzanalysen eine Rekonstruktion funktioneller Netzwerke auf neurophysiologischer Basis. Pilotdaten solcher Auswertungen aus einer aktuellen Doktorarbeit zeigen sehr robuste Ergebnisse, die z.B. Hinweise auf die Anfallspropagation liefern, also die Ausbreitung eines fokal beginnenden Anfalls zu einem sekundär generalisierten Anfall (Ernst, 2016). Die bereits gut etablierte DTI Bildgebung ermöglicht hierzu komplementäre Analysen von strukturellen Netzwerken, innerhalb derer eine solche Propagation am ehesten stattfindet. Die Kombination funktioneller und struktureller Netzwerkanalysen hat ein hohes Potential, individuelle prognostische Faktoren zu liefern, um beispielsweise das Ansprechen auf bestimmte Therapien oder die Erfolgsaussichten eines epilepsiechirurgischen Eingriffes noch besser abzuschätzen. 


\section{Bildgebung bei generalisierten Epilepsien}

Bei generalisierten Epilepsien, die per definitionem das gesamte Gehirn betreffen, spielt die Bildgebung klinisch eine untergeordnete Rolle und dient v.a. dem Ausschluss einer strukturellen Läsion. Eine unauffällige, konventionelle MRT-Untersuchung ist ein Diagnosekriterium, um eine generalisierte Epilepsie zu bestätigen.

Interessanter sind bei generalisierten Epilepsien konzeptionelle, wissenschaftliche Fragen bezüglich der strategischen Bedeutung bestimmter "Relais"-Regionen und die Rolle von funktionellen Netzwerken und Konnektivitätsveränderungen bei der Epileptogenese.

\subsection{Juvenile Myoklonische Epilepsie (JME)}

Der Habilitand hat sich intensiv mit der JME beschäftigt, die nach ihrem Erstbeschreiber auch als Janz-Syndrom bezeichnet wird (Janz and Christian, 1957). JME ist die häufigste generalisierte Epilepsie des Erwachsenenalters und manifestiert sich in jugendlichen Jahren durch kurze, bilaterale, proximale Zuckungen der Arme, sogenannte Myoklonien. Meist erst einige Jahre später kommt es dann auch zu generalisiert konvulsiven Anfällen. Die JME ist in der Regel gut behandelbar und spricht meist gut auf medikamentöse Therapie an.

Auffällig ist ein spezifisches, neuropsychologisches Profil von JME-Patienten (Pascalicchio et al., 2007; Wandschneider et al., 2013) das mit diskreten strukturellen Veränderungen im Frontallappen in Zusammenhang gebracht wurde (Wandschneider et al., 2012).

\subsection{Strukturelle MRT bei JME}

Bildgebende Untersuchungen mit voxel-basierter Morphometrie haben wiederholt solche diskreten Veränderungen gezeigt (Woermann et al., 1999; O'Muircheartaigh et al., 2011). Die größten Überschneidungen der verschiedenen Arbeiten ergaben sich in einer spezifischen Region, dem supplementär motorischen Areal (SMA) wo wiederholt eine Vermehrung der grauen Substanz beschrieben wurde. Im SMA konnten später auch in DTI Studien signifikante Veränderungen bei JME nachgewiesen werden (Vulliemoz et al., 2011). Die funktionelle Konsequenz dieser oft replizierten, diskreten Veränderungen blieb jedoch unklar.

\subsection{Funktionelle MRT bei JME}

Hierfür wurde eine fMRT Studie mit einem kognitiven Paradigma in verschiedenen Schwierigkeitsgraden durchgeführt. Während des sogenannten „Dot-Back“ Paradigmas mussten Probanden in der Kontrollbedingung die Position am Bildschirm auftauchender mit einem Joystick anzeigen. Während der "1-back“ Bedingung mussten sie sich die Position des letzten Punktes merken, und bei jeder Präsentation eines Punktes die Position des vorherigen Punktes anzeigen. Während der „2-back“ Bedingung musste die Position des vorletzten Punktes erinnert und angezeigt werden.

Dies führte zu einer Aktivierung im motorischen System linksseitig durch Bedienung des Joysticks mit der rechten Hand sowie in den 1-back und 2-back Bedingungen zusätzlich zu Aktivierung bilateraler frontaler und parietaler Regionen, die das Arbeitsgedächtnis Netzwerk reflektieren. 
Im Vergleich zu gesunden Kontrollpersonen zeigte sich bei JME Patienten eine erhöhte Aktivierung des motorischen Systems, nur unter der zusätzlichen Arbeitsgedächtnis-Leistung der 1-back und 2back Bedingungen (Vollmar et al., 2011).

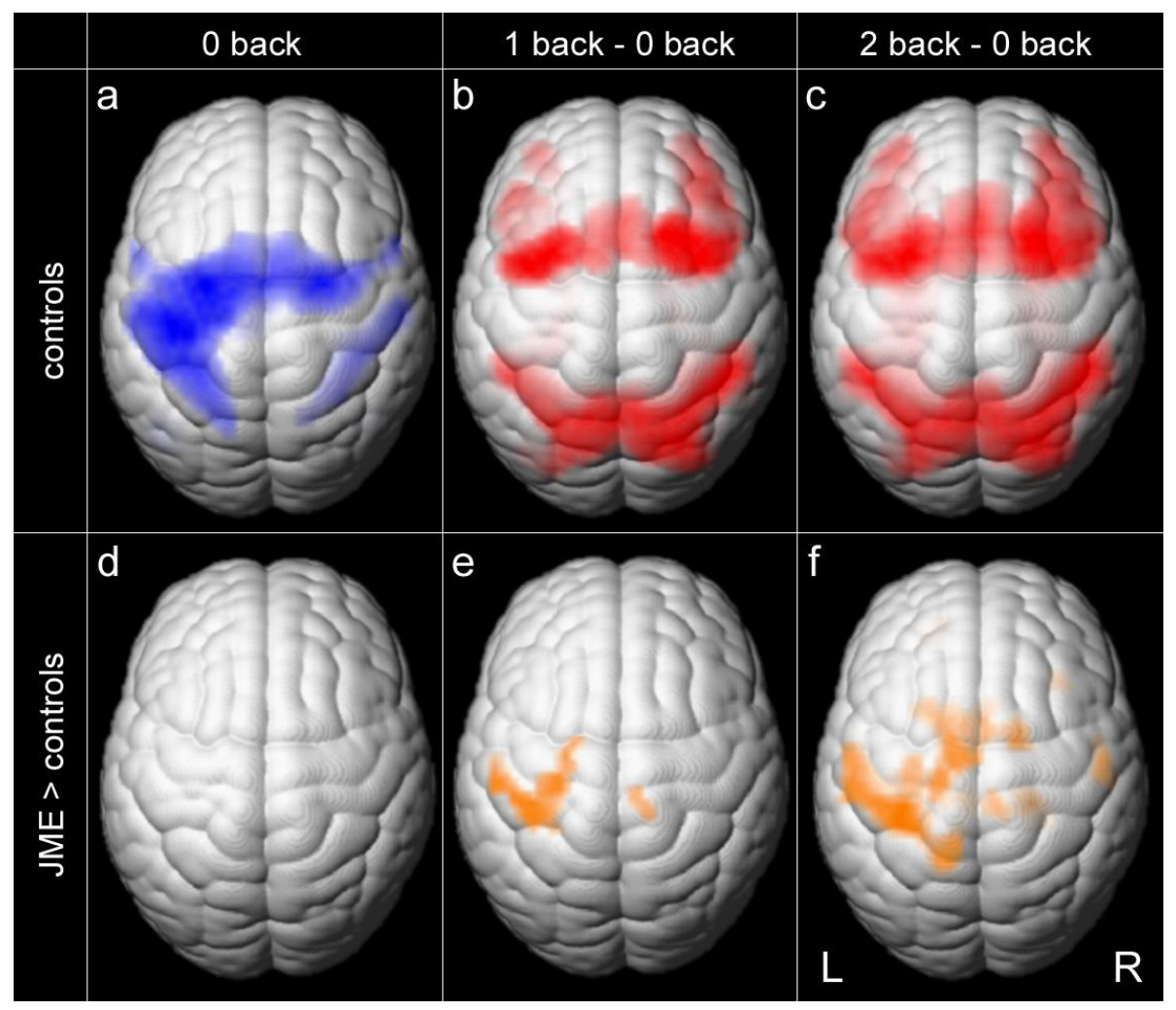

Abbildung 11: fMRT bei gesunden Kontrollen und JME Patienten. Die einfache Lokalisation des präsentierten Punktes mittels Joystick führt zu einer Aktivierung des motorischen Systems (a, blau, linker Gyrus präzenralis und SMA beidseits). In den Arbeitsgedächtnis-Aufgaben 1-back und 2-back zeigt sich nach Subtraktion der motorischen Antwort eine zusätzliche Aktivierung des bilateralen fronto-parietalen Arbeitsgedächtnis-Netzwerkes (b, c, rot). Nur in diesen schwierigen Bedingugen kam es bei JME Patienten zu einer zunehmenden, erhöhten Koaktivierung des motorischen Systems (e,f, orange).

Dass dieser Effekt bei einer einfachen Aufgabe noch nicht sichtbar war, sondern erst bei einer höheren kognitiven Belastung auftrat, ist interessant, da einige Patienten mit JME berichten, dass ihre motorischen Anfälle oft unter besonderer kognitiver Anstrengung auftreten, z.B. in einer Prüfungssituation.

\subsection{Funktionelle Konnektivität bei JME}

Die relativ hohe zeitliche Auflösung der fMRT erlaubt es, für jede Region einen Kurvenverlauf der Signalintensität zu extrahieren. Die Stärke der Korrelation dieser Signalverläufe zwischen verschiedenen Hirnregionen kann als $\mathrm{Maß}$ für die funktionelle Konnektivität dieser Regionen verwendet werden. Bei JME Patienten zeigte sich hier im Vergleich zu gesunden Kontrollen eine erhöhte funktionelle Konnektivität zwischen präfrontalen, kognitiven Arealen und dem motorischen System (Vollmar et al., 2011)

Auch diese Hyperkonnektivität bei JME-Patienten deckt sich gut mit einer weiteren klinischen Beobachtung, der sogenannten „Praxis-Induktion“ von Anfällen (Yacubian and Wolf, 2014). Praxis beschreibt hier die Kombination einer kognitiven Aufgabe mit komplexen, manuellen Tätigkeiten beispielsweise beim Spielen von Instrumenten, Schachspielen, oder, wie von einer Patientin geschildert, dem Zusammenbau von Ikea-Möbeln. 
Vor diesem Hintergrund bietet die mittels fMRT nachgewiesene Hyperkonnektivität eine mögliche Erklärung für den spezifischen Effekt der Praxis-Induktion: einen funktionellen "Kurzschluss“ zwischen dem motorischen System und kognitiven Netzwerken des Frontallappens. Bei gleichzeitiger Aktivierung dieser beiden Systeme kann es offenbar bei JME durch die erhöhte Konnektivität zu ungünstigen Rückkopplungen und einer Übererregbarkeit kommen, die dann zu einem motorischen Anfall führt.

Eine solche pathologisch erhöhte funktionelle Konnektivität wirft die Frage nach möglichen strukturellen Korrelat auf. Eine mögliche Relais-Station, die das präfrontale kognitive und das motorische System verbindet, ist das supplementär motorische Areal (SMA).

\subsection{DTI und strukturelle Konnektivität bei JME}

Eine darauf durchgeführte DTI Studie nahm eine Parzellierung des SMA auf der Basis seiner strukturellen Konnektivität zu übrigen Hirnarealen vor. Hierfür wurde eine Region im Gyrus frontalis superior definiert, von der aus mittels DTI Tracking, die Konnektivität jedes Bildpunktes zu den übrigen Hirnarealen bestimmt wurde. Mittels eines Clustering Algorithmus wurde die Region dann in zwei Subregionen aufgeteilt, die sich durch ihr unterschiedliches Konnektivitätsprofil unterscheiden. So konnte eine rein konnektivitätsbasierte Unterscheidung des posterior gelegenen SMA von anterior angrenzenden kognitiven Arealen erfolgen. Im nächsten Schritte wurde dann die Konnektivität beider Subregionen untersucht.

Hier zeigte sich im Gruppenvergleich bei JME Patienten ebenfalls eine erhöhte strukturelle Konnektivität zwischen präfrontalen, kognitiven Arealen und dem motorischen System, die signifikant mit der in der fMRT Studie beobachteten funktionellen Konnektivität korreliert war (Vollmar et al., 2012). Damit erhärteten die Befunde der DTI Konnektivität die zuvor beobachteten funktionellen Effekte und konnten zeigen, dass diesen messbare strukturelle Veränderungen zugrunde liegen.

Neben diesen direkten, kortiko-kortikalen Konnektivitätsveränderungen zeigte sich bei JMEPatienten in einer weiteren DTI Studie auch eine spezifische Reduktion der anterioren thalamokortikalen Konnektivität zum frontalen Kortex (O'Muircheartaigh et al., 2012). Auch dies fügt sich gut in das Gesamtbild, da der Thalamus über ebendiese thalamofrontalen Verbindungen potentiell einen inhibitorischen Effekt auf den frontalen Kortex ausüben könnte - die aber bei der JME reduziert sind.

\subsection{Untersuchungen an gesunden Geschwistern}

Nach der Beschreibung solcher Veränderungen bei Patienten mit chronischer Epilepsie stellt sich immer die Frage, ob die beobachteten Veränderungen die Ursache oder eine sekundäre Folge der Epilepsie, oder möglicherweise eine Folge anderer Faktoren, wie der Medikation, sind. Um dies zu klären, wurden im Verlauf die gleichen Untersuchungen an gesunden Geschwistern von JMEPatienten durchgeführt. Hier zeigte sich, dass die gleichen Konnektivitätsveränderungen auch bei gesunden Geschwistern nachweisbar waren, die keine JME hatten, niemals einen epileptischen Anfall hatten und keine Medikation einnahmen (Wandschneider et al., 2014). Damit war klar, dass es sich bei der "Hyperkonnektivität" des motorischen Systems um eine genetisch determinierte Prädisposition handelt, die bei gesunden Geschwistern ebenfalls nachgewiesen werden kann und die in Kombination mit anderen Faktoren bei JME-Patienten zur klinischen Manifestation einer Epilepsie führen kann. Dies illustriert insgesamt die sehr multifaktorielle Ätiologie einer Erkrankung wie der JME, bei der mehrere Faktoren zusammentreffen müssen, damit sich die Erkrankung manifestiert. 
In der Familie eines JME-Patienten konnten wir beispielsweise eine bis dato unbekannte Mutation im Gephyrin-Gen nachweisen, die zu einem veränderten Arrangement von inhibitorischen GABARezeptoren führt (Dejanovic et al., 2014). Der Vater des Patienten war ebenfalls Träger dieser Mutation, war jedoch gesund. Interessanterweise konnte die transkranielle Magnetstimulation mit Doppel-Puls beim Patienten und seinem Vater eine gestörte kortikale Inhibition nachweisen, d.h. auch das Vorliegen messbarer neurophysiologischer Veränderungen reicht noch nicht aus, um sich klinisch als Epilepsie zu manifestieren.

\subsection{Zusammenfassung Bildgebung bei JME}

Zusammenfassend ergibt sich damit folgende Befundkonstellation der Bildgebung bei JME (Wandschneider and Vollmar, 2018) (Abb.12):

1. Morphometrische Analysen der strukturellen MRT zeigen ein erhöhtes Volumen grauer Substanz im SMA. In diesem Kontext ist es wichtig, dass die Abnahme des initialen Volumens von grauer Substanz im Kindes- und Jugendalter einen wichtigen Reifungsprozess der Hirnentwicklung darstellt. Dies geht einher mit der Elimination redundanter Synapsen („synaptic pruning“) und ist mit der Entwicklung kognitiver Funktionen korreliert. Das erhöhte Volumen grauer Substanz kann hier also eine gestörte Entwicklung mit verminderter Rückbildung redundanter Synapsen reflektieren. Viele morphometrische Studien zeigten auch eine Verminderung grauer Substanz im Thalamus.

2. Funktionelle Studien mittels fMRT habe eine vermehrte Koaktivierung von motorischen Kortex während kognitiver Anstrengung gezeigt. Außerdem zeigte sich eine erhöhte funktionelle Konnektivität zwischen dem motorischen System und präfrontalen kognitiven Arealen. Diese Hyperkonnektivität könnte eine Folge mangelnder Rückbildung von Synapsen sein und erklärt außerdem plausibel den Mechanismus der Praxis-Induktion bei JME.

3. DTI Konnektivitätsanalysen haben gezeigt, dass der erhöhten funktionellen Konnektivität zwischen motorischen und kognitiven Arealen ein strukturelles Korrelat zugrunde liegt. Außerdem zeigte sich eine Verminderung thalamofrontaler Verbindungen, die einen möglichen inhibitorischen Effekt des Thalamus auf die hyperkonnektierten und übererregbaren frontalen Areale einschränken könnte.

4. Untersuchungen bei Familienangehörigen von JME Patienten haben gezeigt, dass diese messbaren Veränderungen genetisch determiniert sind und nicht eine sekundäre Folge der chronischen Epilepsie darstellen. Gleichzeitig illustrieren diese Befunde bei gesunden Geschwistern aber auch, dass die in der Bildgebung identifizierbaren Veränderungen allein noch nicht zu einer Erkrankung führen. Weitere, aktuell noch unbekannte Faktoren müssen hinzukommen, damit sich auf dem Boden der hier beschriebenen Veränderungen klinisch eine JME manifestiert. 


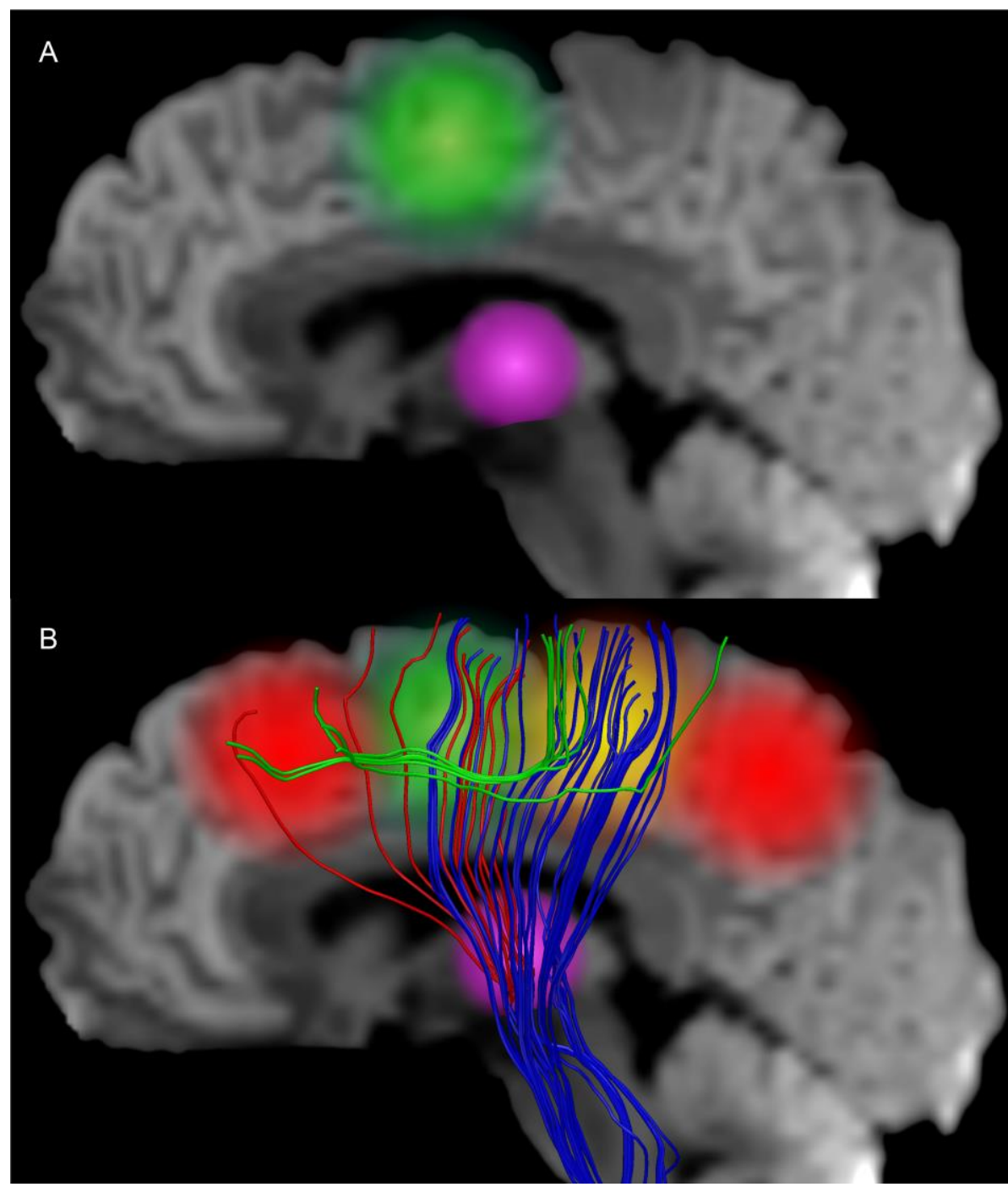

Abbildung 12: Zusammenfassung bildgebender Befunde bei JME. A) Die beiden am häufigsten replizierten Befunde aus morphometrischen MRT Studien bei JME sind eine Vermehrung von grauer Substanz im supplementär motorischen Areal (grün) und eine Reduktion im Thalamus (pink). B) Die in Abb.11 beschriebene funktionelle Co-Aktivierung des motorischen Systems (orange) bei kognitiver Aktivierung (rot) werden durch eine bei JME erhöhte strukturelle Konnektivität zwischen präfrontalen kognitiven Arealen und dem motorischen System vermittelt (grüne Verbindungen). Diese durchkreuzen die ansonsten in dieser Region dominierenden absteigenden motorischen Bahnen (blaue Verbindungen), was Befunde mit einer reduzierten fraktionellen Anisotropie in DTI Daten erklärt. Die reduzierten thalamo-kortikalen Verbindungen zum Frontallappen (rote Verbindungen) erschweren zusätzlich eine hemmende Rückkopplung auf die hyperkonnektierten und übererregbaren kognitiven und motorischen Systeme. 


\subsection{Ausblicke - Bildgebung bei JME}

Wie oben erwähnt, ist die JME die häufigste generalisierte Epilepsieform bei Erwachsenen. In dieser großen Gruppe von Patienten lassen sich klinisch durchaus weitere Unterscheidungen treffen. Insbesondere Triggerfaktoren, die Anfälle auslösen, sind nicht bei allen Patienten gleich. Bei einer Subgruppe von Patienten besteht die o.g. Praxis-Induktion von Anfällen, bei anderen liegt dagegen eine Photosensibilität vor, d.h. Anfälle können durch flackernde Lichter ausgelöst werden. Wieder andere Patienten berichten von Anfällen unter besonderer, emotionaler Belastung. Nach gezielter Rekrutierung von Patienten mit solchen spezifischen Triggerfaktoren könnte mit Hilfe von Konnektivitätsanalysen untersucht werden, ob sich Subgruppen der JME anhand von spezifischen Netzwerk- und Konnektivitätsprofilen unterscheiden lassen, beispielsweise durch eine erhöhte Konnektivität zwischen dem visuellen und dem motorischen System bei photosensiblen Patienten. Dies würde weitere pathophysiologische Mechanismen erklären und könnte eine individuelle Risikoabschätzung für bestimmte, anfallsauslösende Faktoren ermöglichen. 


\section{Zusammenfassung}

In der bildgebenden Diagnostik der fokalen Epilepsien steht die Anwendung beim einzelnen Patienten im Rahmen der prächirurgischen Evaluation im Vordergrund. Hier ist eine qualitativ gute, gezielt durchgeführte MRT-Untersuchung der wichtigste Baustein, der jedoch in vielen Fällen von einem weiten Spektrum komplementärer bildgebender Methoden ergänzt werden muss, um eine sichere Identifikation der epileptogenen Zone zu ermöglichen. Die multimodale Bildverarbeitung ermöglicht hierbei eine konklusive Beurteilung aller verfügbaren Informationen und erhöht dadurch die diagnostische Sicherheit. Außerdem erlaubt sie eine detaillierte individuelle Planung operativer Eingriffe und hilft intraoperativ, die Ergebnisse der prächirurgischen Evaluation im OP Saal anatomisch exakt umzusetzen. So sind auch in schwierigen Fällen gute Ergebnisse von epilepsiechirurgischen Engriffen möglich.

Bei den generalisierten Epilepsien ist die klinische Rolle der Bildgebung deutlich einfacher und dient im Wesentlichen der Diagnosesicherung durch Ausschluss einer strukturellen Läsion. Die Stärke der Bildgebung liegt hier in der wissenschaftlichen Anwendung zur Aufklärung pathophysiologischer Mechanismen. Im Falle der juvenilen myoklonischen Epilepsie konnte die Bildgebung viele Hinweise beisteuern, die das komplexe Zusammenspiel aus genetischer Anlage, gestörten Entwicklung, Hyperkonnektivität und Übererregbarkeit besser verständlich machen.

In beiden Bereichen deutet sich aber auch an, dass die bisher übliche, dichotome Unterscheidung in fokale und generalisierte Epilepsien durch neue Erkenntnisse ergänzt wird. Bei fokalen Epilepsien gewinnen wir zunehmend Einblicke in die zusätzlichen wirksamen "generalisierten" Netzwerke, die zur Modulation der Erkrankung beitragen. Bei den generalisierten Epilepsien lassen sich dagegen teilweise sehr fokale, präzise lokalisierbare Veränderungen identifizieren, die eher "bilateral frontal“ als wirklich generalisiert sind. Gerade die aktuell möglichen Konnektivitätsanalysen auf der Basis bildgebender Verfahren können hier in den kommenden Jahren weitere Erkenntnisse liefern, die die aktuelle starre Klassifikation bereichern und unser pathophysiologisches Verständnis der Epilepsien verbessern können. 


\section{Literatur}

Bernasconi A, Bernasconi N, Bernhardt BC, Schrader D. Advances in MRI for 'cryptogenic' epilepsies. Nat. Rev. Neurol. 2011; 7: 99-108. DOI 10.1038/nrneurol.2010.199

Bilgin O, Vollmar C, Peraud A, la Fougere C, Beleza P, Noachtar S. Ictal SPECT in Sturge-Weber syndrome. Epilepsy Res. 2008; 78: 240-3. DOI 10.1016/j.eplepsyres.2007.12.006

de Boer HM, Mula M, Sander JW. The global burden and stigma of epilepsy. Epilepsy Behav. 2008; 12: 540-546. DOI 10.1016/j.yebeh.2007.12.019

Bonelli SB, Thompson PJ, Yogarajah M, Vollmar C, Powell RHW, Symms MR, et al. Imaging language networks before and after anterior temporal lobe resection: results of a longitudinal fMRI study. Epilepsia 2012; 53: 639-50. DOI 10.1111/j.1528-1167.2012.03433.x

Borggraefe I, Catarino CB, Rémi J, Vollmar C, Peraud A, Winkler PA, et al. Lateralization of cortical negative motor areas. Clin. Neurophysiol. 2016; 127: 3314-21. DOI 10.1016/j.clinph.2016.08.001

Catarino CB*, Vollmar C*, Küpper C, Seelos K, Gallenmüller C, Bartkiewicz J, et al. Brain diffusion tensor imaging changes in cerebrotendinous xanthomatosis reversed with treatment. J. Neurol. 2018; 265:388-93. DOI 10.1007/s00415-017-8711-9

Catarino CB, Vollmar C, Noachtar S. Paradoxical lateralization of non-invasive electroencephalographic ictal patterns in extra-temporal epilepsies. Epilepsy Res. 2012; 99: 147-55. DOI 10.1016/j.eplepsyres.2011.11.002

Centeno M, Vollmar C, Stretton J, Symms MR, Thompson PJ, Richardson MP, et al. Structural changes in the temporal lobe and piriform cortex in frontal lobe epilepsy. Epilepsy Res. 2014; 108: 978-81. DOI 10.1016/j.eplepsyres.2014.03.001

Centeno M*, Vollmar C*, O'Muircheartaigh J, Stretton J, Bonelli S, Symms MR, Barker GJ, Kumari V, Thompson PJ, Duncan JS, Richardson MP. Koepp MJ. Memory in frontal lobe epilepsy: an fMRI study. Epilepsia 2012; 53: 1756-64. DOI 10.1111/j.1528-1167.2012.03570.x

Centeno M, Koepp MJMJ, Vollmar C, Stretton J, Sidhu M, Michallef C, et al. Language dominance assessment in a bilingual population: validity of $\mathrm{fMRI}$ in the second language. Epilepsia 2014; 55: 1504-11. DOI 10.1111/epi.12757

Cloppenborg T, May TW, Blümcke I, Grewe P, Hopf LJ, Kalbhenn T, et al. Trends in epilepsy surgery: stable surgical numbers despite increasing presurgical volumes. J. Neurol. Neurosurg. Psychiatry 2016; 87: 1322-1329. DOI 10.1136/jnnp-2016-313831

Cunha JPS, Choupina HMP, Rocha AP, Fernandes JM, Achilles F, Loesch AM, Vollmar C, Hartl E, Noachtar S. NeuroKinect: A Novel Low-Cost 3Dvideo-EEG System for Epileptic Seizure Motion Quantification. PLoS One 2016; 11: e0145669. DOI 10.1371/journal.pone.0145669

Dejanovic B, Lal D, Catarino CB, Arjune S, Belaidi AA, Trucks H, Vollmar C, et al. Exonic microdeletions of the gephyrin gene impair GABAergic synaptic inhibition in patients with idiopathic generalized epilepsy. Neurobiol. Dis. 2014; 67: 88-96. DOI 10.1016/j.nbd.2014.02.001

Duncan JS, Winston GP, Koepp MJ, Ourselin S. Brain imaging in the assessment for epilepsy surgery. Lancet. Neurol. 2016; 15: 420-33. DOI 10.1016/S1474-4422(15)00383-X

Feddersen B, Vollmar C, Rémi J, Stephan T, Flanagin VL, Noachtar S. Seizures From Solving Sudoku Puzzles. JAMA Neurol. 2015; 72: 1524-6. DOI 10.1001/jamaneurol.2015.2828

Goc J, Khalilieh N, Hartl E, Noachtar S, Vollmar C. Quantitative analysis of fiber density from Diffusion 
Tensor Imaging identifies structural changes in cryptogenic focal epilepsy. Abstract. American Epilepsy Society Annual Meeting 2014; www.aesnet.org, ID 1868721.

Guldiken B, Vollmar C, Peraud A, Noachtar S. Intraoperative electrical stimulation of the cortex reveals the speech area missed by extraoperative stimulation because of epicortical hematoma. Acta Neurochir. (Wien). 2015; 157: 1585-6. DOI 10.1007/s00701-015-2517-2

Hartl E, Rémi J, Vollmar C, Goc J, Loesch AM, Rominger A, et al. PET imaging in extratemporal epilepsy requires consideration of electroclinical findings. Epilepsy Res. 2016; 125: 72-76. DOI 10.1016/j.eplepsyres.2016.05.010

Janz D, Christian W. Impulsive Petit mal. Dtsch Z Nervenheilk 1957; 176: 348-386. DOI $10.1007 / \mathrm{bf00242439}$

Kovac S, Scott C, Rugg-Gunn F, Miserocchi A, Vollmar C, Rodionov R, et al. Unusual cortical stimulation findings: connectivity between primary motor and supplementary motor areas. Epilepsy Behav. 2010; 19: 639-42. DOI 10.1016/j.yebeh.2010.09.006

Loesch AM, Feddersen B, Tezer FI, Hartl E, Rémi J, Vollmar C, et al. Seizure semiology identifies patients with bilateral temporal lobe epilepsy. Epilepsy Res. 2015; 109: 197-202. DOI 10.1016/j.eplepsyres.2014.11.002

Loesch AM, Steger H, Losher C, Hartl E, Rémi J, Vollmar C, et al. Seizure-associated aphasia has good lateralizing but poor localizing significance. Epilepsia 2017; 58: 1551-1555. DOI 10.1111/epi.13835

O'Muircheartaigh J, Vollmar C, Barker GJ, Kumari V, Symms MR, Thompson P, et al. Abnormal thalamocortical structural and functional connectivity in juvenile myoclonic epilepsy. Brain 2012; 135: 3635-44. DOI 10.1093/brain/aws296

O'Muircheartaigh J, Vollmar C, Barker GJ, Kumari V, Symms MR, Thompson P, et al. Focal structural changes and cognitive dysfunction in juvenile myoclonic epilepsy. Neurology 2011; 76: 34-40. DOI 10.1212/WNL.0b013e318203e93d

Von Oertzen J, Urbach H, Jungbluth S, Kurthen M, Reuber M, Fernández G, et al. Standard magnetic resonance imaging is inadequate for patients with refractory focal epilepsy. J. Neurol. Neurosurg. Psychiatry 2002; 73: 643-7. DOI 10.1136/jnnp.73.6.643

Pascalicchio TF, de Araujo Filho GM, da Silva Noffs MH, Lin K, Caboclo LOSF, Vidal-Dourado M, et al. Neuropsychological profile of patients with juvenile myoclonic epilepsy: a controlled study of 50 patients. Epilepsy Behav. 2007; 10: 263-7. DOI 10.1016/j.yebeh.2006.11.012

Pfluger T, Vollmar C, Wismüller A, Dresel S, Berger F, Suntheim P, et al. Quantitative comparison of automatic and interactive methods for MRI-SPECT image registration of the brain based on 3dimensional calculation of error. J. Nucl. Med. 2000; 41: 1823-9.

Rémi J*, Vollmar C*, de Marinis A, Heinlin J, Peraud A, Noachtar S. Congruence and discrepancy of interictal and ictal EEG with MRI lesions in focal epilepsies. Neurology 2011; 77: 1383-90. DOI 10.1212/WNL.0b013e31823152c3

Rémi J, Wagner P, O'Dwyer R, Silva Cunha JP, Vollmar C, Krotofil I, et al. Ictal head turning in frontal and temporal lobe epilepsy. Epilepsia 2011; 52: 1447-51. DOI 10.1111/j.1528-1167.2011.03076.x

Rodionov R, Vollmar C, Nowell M, Miserocchi A, Wehner T, Micallef C, et al. Feasibility of multimodal 3D neuroimaging to guide implantation of intracranial EEG electrodes. Epilepsy Res. 2013; 107: 91100. DOI 10.1016/j.eplepsyres.2013.08.002

Ryvlin P, Cross JH, Rheims S. Epilepsy surgery in children and adults. Lancet. Neurol. 2014; 13: 11141126. DOI 10.1016/S1474-4422(14)70156-5

Sidhu MK, Stretton J, Winston GP, Bonelli S, Centeno M, Vollmar C, et al. A functional magnetic 
resonance imaging study mapping the episodic memory encoding network in temporal lobe epilepsy. Brain 2013; 136: 1868-88. DOI 10.1093/brain/awt099

Silva Cunha JP, Rémi J, Vollmar C, Fernandes JM, Gonzalez-Victores JA, Noachtar S. Upper limb automatisms differ quantitatively in temporal and frontal lobe epilepsies. Epilepsy Behav. 2013; 27: 404-8. DOI 10.1016/j.yebeh.2013.02.026

So EL, Lee RW. Epilepsy surgery in MRI-negative epilepsies. Curr. Opin. Neurol. 2014; 27: 206-212. DOI 10.1097/WCO.0000000000000078

Stretton J, Winston G, Sidhu M, Centeno M, Vollmar C, Bonelli S, et al. Neural correlates of working memory in Temporal Lobe Epilepsy--an fMRI study. Neuroimage 2012; 60: 1696-703. DOI 10.1016/j.neuroimage.2012.01.126

Thorsteinsdottir J*, Vollmar C*, Tonn JC, Kreth FW, Noachtar S, Peraud A. Good outcome after individualized SEEG implantation and navigated resection in patients with lesional and non-lesional focal epilepsy. submitted 2018

Vollmar C, Noachtar S. Importance of imaging diagnostics and EEG for differential diagnosis of epileptic seizures. Nervenarzt 2017; 88: 1119-1125. DOI 10.1007/s00115-017-0402-3

Vollmar C, Goc J, Hartl E, Noachtar S,. U-Fiber Track Density Imaging identifies specific alterations in the epileptogenic zone in individual patients with cryptogenic focal epilepsy. Abstract. American Epilepsy Society Annual Meeting 2016; www.aesnet.org, ID 195991.

Vollmar C. Multimodale Bildgebung in der prächirurgischen Epilepsiediagnostik. Zeitschrift für Epileptol. 2012; 25: 175-181. DOI 10.1007/s10309-012-0256-4

Vollmar C, O'Muircheartaigh J, Symms MR, Barker GJ, Thompson P, Kumari V, et al. Altered microstructural connectivity in juvenile myoclonic epilepsy: the missing link. Neurology 2012; 78: 1555-9. DOI 10.1212/WNL.0b013e3182563b44

Vollmar C, la Fougère C. [Neuroimaging of epilepsies]. Nervenarzt 2012; 83: 167-71. DOI 10.1007/s00115-011-3334-3

Vollmar C, O'Muircheartaigh J, Barker GJ, Symms MR, Thompson P, Kumari V, et al. Motor system hyperconnectivity in juvenile myoclonic epilepsy: a cognitive functional magnetic resonance imaging study. Brain 2011; 134: 1710-9. DOI 10.1093/brain/awr098

Vollmar C, Diehl B. Diffusion tensor tractography in extratemporal lobe epilepsy. In: Koubeissi MZ, Maciunas RJ, editor(s). Extratemporal Lobe Epilepsy Surgery. John Libbey; 2011. p. 197-205. ISBN-10: 2742007725

Vollmar C, O'Muircheartaigh J, Barker GJ, Symms MR, Thompson P, Kumari V, et al. Identical, but not the same: intra-site and inter-site reproducibility of fractional anisotropy measures on two 3.0T scanners. Neuroimage 2010; 51: 1384-94. DOI 10.1016/j.neuroimage.2010.03.046

Vollmar C, Noachtar S, Winkler PA. Multimodal image processing in pre-surgical planning. In: Lüders HO, editor. Textbook of Epilepsy Surgery. London: Informa UK Ltd; 2008. p. 771-777. DOI 10.3109/9780203091708-100

Vollmar C*, Feddersen B*, Beckmann BM, Kääb S, Noachtar S. Seizures on hearing the alarm clock. Lancet 2007; 370: 2172. DOI 10.1016/S0140-6736(07)61913-8

Vollmar C, Noachtar S. Neuroimaging in epilepsy. Turkish J. Neurol. 2004; 10: 185-200.

Vulliemoz S, Vollmar C, Koepp MJ, Yogarajah M, O’Muircheartaigh J, Carmichael DW, et al. Connectivity of the supplementary motor area in juvenile myoclonic epilepsy and frontal lobe epilepsy. Epilepsia 2011; 52: 507-14. DOI 10.1111/j.1528-1167.2010.02770.x 
Wandschneider B, Vollmar C. Besondere bildgebende Befunde bei primär generalisierten Epilepsien. Zeitschrift für Epileptol. 2018; 31: 144-47. DOI 10.1007/s10309-018-0179-9

Wandschneider B, Centeno M, Vollmar C, Symms M, Thompson PJ, Duncan JS, et al. Motor coactivation in siblings of patients with juvenile myoclonic epilepsy: an imaging endophenotype? Brain 2014; 137: 2469-79. DOI 10.1093/brain/awu175

Wandschneider B, Centeno M, Vollmar C, Stretton J, O'Muircheartaigh J, Thompson PJ, et al. Risktaking behavior in juvenile myoclonic epilepsy. Epilepsia 2013; 54: 2158-65. DOI 10.1111/epi.12413

Wandschneider B, Thompson PJ, Vollmar C, Koepp MJ. Frontal lobe function and structure in juvenile myoclonic epilepsy: a comprehensive review of neuropsychological and imaging data. Epilepsia 2012; 53: 2091-8. DOI 10.1111/epi.12003

West S, Nolan SJ, Newton R. Seminar in Epileptology Surgery for epilepsy : a systematic review. Epileptic Disord. 2016; 18: 113-121. DOI 10.1684/epd.2016.0825

Wiebe S, Blume WT, Girvin JP, Eliasziw M. A randomized, controlled trial of surgery for temporal-lobe epilepsy. N. Engl. J. Med. 2001; 345: 311-318. DOI 10.1056/NEJM200108023450501

Winkler PA, Vollmar C, Krishnan KG, Pfluger T, Brückmann H, Noachtar S. Usefulness of 3-D reconstructed images of the human cerebral cortex for localization of subdural electrodes in epilepsy surgery. Epilepsy Res. 2000; 41: 169-178. DOI 10.1016/S0920-1211(00)00137-6

Woermann FG, Free SL, Koepp MJ, Ashburner J, Duncan JS. Voxel-by-voxel comparison of automatically segmented cerebral gray matter--A rater-independent comparison of structural MRI in patients with epilepsy. Neuroimage 1999; 10: 373-84. DOI 10.1006/nimg.1999.0481

Woermann FG, Vollmar C. Clinical MRI in children and adults with focal epilepsy: a critical review. Epilepsy Behav. 2009; 15: 40-9. DOI 10.1016/j.yebeh.2009.02.032

Yacubian EM, Wolf P. Praxis induction. Definition, relation to epilepsy syndromes, nosological and prognostic significance. A focused review. Seizure 2014; 23: 247-251. DOI 10.1016/j.seizure.2014.01.011

Yasuda CL, Centeno M, Vollmar C, Stretton J, Symms M, Cendes F, et al. The effect of topiramate on cognitive fMRI. Epilepsy Res. 2013; 105: 250-5. DOI 10.1016/j.eplepsyres.2012.12.007

Yogarajah M, Focke NK, Bonelli SB, Thompson P, Vollmar C, McEvoy AW, et al. The structural plasticity of white matter networks following anterior temporal lobe resection. Brain 2010; 133: 2348-64. DOI 10.1093/brain/awq175

Yogarajah M, Focke NK, Bonelli S, Cercignani M, Acheson J, Parker GJM, et al. Defining Meyer's looptemporal lobe resections, visual field deficits and diffusion tensor tractography. Brain 2009; 132: 1656-68. DOI 10.1093/brain/awp114

\footnotetext{
* Beide Autoren haben zu gleichen Teilen beigetragen
} 


\section{Danksagung}

Im Laufe meiner Ausbildung und wissenschaftlichen Tätigkeit durfte ich mit vielen Kollegen zusammenarbeiten denen mein Dank gebührt.

Bei Prof. T. Pfluger und Prof. K. Hahn begann ich meine wissenschaftliche Laufbahn im Rahmen der Doktorarbeit in der Klinik für Nuklearmedizin und danke beiden für die ersten Einblicke in wissenschaftliches Arbeiten, die Begeisterung für die Bildgebung und die Möglichkeit eigene Interessen auszubauen. Prof. P. Winkler danke ich für den Kontakt zur Epileptologie, die Ermutigung zur Entwicklung neurochirurgisch relevanter bildgebenden Verfahren und die Begeisterung für die Neuroanatomie.

Nach meinem Wechsel in die Neurologische Klinik hatte ich bei Prof. S. Noachtar, unter der Klinikleitung von Prof. T. Brand, Gelegenheit diagnostische Methoden weiterzuentwickeln und unmittelbar klinisch anzuwenden. Ich danke ihm für diese Möglichkeit, die langjährige Freundschaft, die epileptologische Ausbildung und die Inspiration durch seine herausragend strukturierte Herangehensweise an klinische und wissenschaftliche Fragestellungen.

Während eines knapp vierjährigen Forschungsaufenthaltes in London, am UCL Institute of Neurology, Queen Square, hatte ich die Möglichkeit mich intensiv auf die Forschung zu konzentrieren, neue bildgebende Methoden fundiert zu erlernen und die Vorzüge eines exzellenten Wissenschaftsstandortes zu nutzen. Meinen Betreuern, Prof. M. Koepp, Dr. M. Symms und Kollegen wie Prof. J. Duncan, Prof. B. Diehl, Dr. J. O'Muircheartaigh, Dr. S. Bonelli, Dr. M. Yogarajah, Dr. R. Rodionov, Dr. B. Wandschneider und vielen anderen danke ich für die großartige klinische und wissenschaftliche Zusammenarbeit, die freundschaftliche Aufnahme und die Einblicke in britische Medizin, Forschung und Lebenskultur. Dem Wellcome Trust danke ich für die Finanzierung dieses Projektes.

Nach der Rückkehr nach München durfte ich meine Tätigkeit in Prof. Noachtar's EpilepsieArbeitsgruppe fortsetzen, jetzt unter der Klinikleitung von Frau Prof. M. Dieterich, der ich für diese Möglichkeit, die weitere Unterstützung und die Annahme als Habilitand danke.

Den Kollegen der Epilepsie-Arbeitsgruppe Prof. B. Feddersen, PD Dr. J. Remi, Dr. S. Weil, Dr. S. Arnold, Dr. C. Stoyke, Dr. C. Catarino, Dr. E. Hartl, Dr. AM Lösch, Dr. J. Bartkiewicz, Dr. H. Lohr, Dr. K. Ernst und allen Doktoranden danke ich für die stets gute Zusammenarbeit in freundschaftlicher Atmosphäre.

In der Neurochirurgischen Klinik danke ich insbesondere Frau Prof. A. Peraud, Prof. F. Kreth, Dr. M. Kunz und Prof. J.-C. Tonn für die hervorragende langjährige Kooperation bei der gemeinsamen Behandlung unserer Patienten.

Prof. P. Bartenstein, dem jetzigen Leiter der Klinik für Nuklearmedizin, danke ich für die gute klinische Zusammenarbeit in der Diagnostik unserer Patienten und die Unterstützung im Fachmentorat.

Prof. H. Brückmann, Dr. K. Seelos und allen anderen Kollegen der Abteilung für Neuroradiologie danke ich für die herzliche Aufnahme in die Abteilung und die neuroradiologische Weiterbildung.

PD Dr. I. Borggräfe aus dem Dr. von Hauner'schen Kinderspital danke ich für die gute Zusammenarbeit bei der gemeinsamen Behandlung pädiatrischer Epilepsie-Patienten.

Prof. J. Silva Cunha von der Universität Porto und Prof. N. Navab vom Lehrstuhl für Informatik der Technischen Universität München danke ich für die gute Zusammenarbeit an innovativen, technisch herausfordernden Projekten. 


\section{7 Übersicht der relevanten Fachpublikationen}

- Catarino CB*, Vollmar C*, Küpper C, Seelos K, Gallenmüller C, Bartkiewicz J, et al. Brain diffusion tensor imaging changes in cerebrotendinous xanthomatosis reversed with treatment. J. Neurol. 2017; 265: 388-93.

- Feddersen B, Vollmar C, Rémi J, Stephan T, Flanagin VL, Noachtar S. Seizures From Solving Sudoku Puzzles. JAMA Neurol. 2015; 72: 1524-6.

- O'Muircheartaigh J, Vollmar C, Barker GJ, Kumari V, Symms MR, Thompson P, et al. Abnormal thalamocortical structural and functional connectivity in juvenile myoclonic epilepsy. Brain 2012; 135: 3635-44.

- O'Muircheartaigh J, Vollmar C, Barker GJ, Kumari V, Symms MR, Thompson P, et al. Focal structural changes and cognitive dysfunction in juvenile myoclonic epilepsy. Neurology 2011; 76: 34-40.

- Pfluger T, Vollmar C, Wismüller A, Dresel S, Berger F, Suntheim P, et al. Quantitative comparison of automatic and interactive methods for MRI-SPECT image registration of the brain based on 3dimensional calculation of error. J. Nucl. Med. 2000; 41: 1823-9.

- Rémi J*, Vollmar C*, de Marinis A, Heinlin J, Peraud A, Noachtar S. Congruence and discrepancy of interictal and ictal EEG with MRI lesions in focal epilepsies. Neurology 2011; 77: 1383-90.

- Rodionov R, Vollmar C, Nowell M, Miserocchi A, Wehner T, Micallef C, et al. Feasibility of multimodal 3D neuroimaging to guide implantation of intracranial EEG electrodes. Epilepsy Res. 2013; 107: 91100.

- Vollmar C, Stredl I, Heinig M, Noachtar S, Rémi J. Unilateral temporal interictal epileptiform discharges correctly predict the epileptogenic zone in lesional temporal lobe epilepsy. Epilepsia 2018; 59: 1577-82.

- Vollmar C, Noachtar S. Importance of imaging diagnostics and EEG for differential diagnosis of epileptic seizures. Nervenarzt 2017; 88: 1119-1125.

- Vollmar C. Multimodale Bildgebung in der prächirurgischen Epilepsiediagnostik. Zeitschrift für Epileptol. 2012; 25: 175-181.

- Vollmar C, O'Muircheartaigh J, Symms MR, Barker GJ, Thompson P, Kumari V, et al. Altered microstructural connectivity in juvenile myoclonic epilepsy: the missing link. Neurology 2012; 78: 1555-9.

- Vollmar C, O'Muircheartaigh J, Barker GJ, Symms MR, Thompson P, Kumari V, et al. Motor system hyperconnectivity in juvenile myoclonic epilepsy: a cognitive functional magnetic resonance imaging study. Brain 2011; 134: 1710-9.

- Vollmar C, Diehl B. Diffusion tensor tractography in extratemporal lobe epilepsy. In: Koubeissi MZ, Maciunas RJ, editor(s). Extratemporal Lobe Epilepsy Surgery. John Libbey; 2011. p. 197-205.

- Vollmar C, O'Muircheartaigh J, Barker GJ, Symms MR, Thompson P, Kumari V, et al. Identical, but not the same: intra-site and inter-site reproducibility of fractional anisotropy measures on two 3.0T scanners. Neuroimage 2010; 51: 1384-94.

- Vollmar C, Noachtar S, Winkler PA. Multimodal image processing in pre-surgical planning. In: Lüders HO, editor. Textbook of Epilepsy Surgery. London: Informa UK Ltd; 2008. p. 771-777.

- Wandschneider B, Vollmar C. Besondere bildgebende Befunde bei primär generalisierten Epilepsien. Zeitschrift für Epileptol. 2018

- Winkler PA, Vollmar C, Krishnan KG, Pfluger T, Brückmann H, Noachtar S. Usefulness of 3-D reconstructed images of the human cerebral cortex for localization of subdural electrodes in epilepsy surgery. Epilepsy Res. 2000; 41: 169-178. 